\title{
Dysfunctional cerebrovascular tone contributes to cognitive impairment in a non-obese rat model of prediabetic challenge: Role of suppression of autophagy and modulation by anti-diabetic drugs
}

\section{Walaa Kamel Fakih}

American University of Beirut

Ali Mroueh

American University of Beirut

Houssein F. Salah

American University of Beirut

Ali Eid

American University of Beirut

Makram F. Obeid

American University of Beirut

Firas F. Kobeissy

American University of Beirut

Hala Darwish

American University of Beirut

Ahmed F. El-Yazbi ( $\nabla$ ae88@aub.edu.lb )

American University of Beirut https://orcid.org/0000-0003-3432-3038

\section{Research}

Keywords: Prediabetes, cerebral artery tone, cognitive impairment, hippocampal inflammation

Posted Date: December 23rd, 2019

DOI: https://doi.org/10.21203/rs.2.19454/v1

License: (c) (i) This work is licensed under a Creative Commons Attribution 4.0 International License. Read Full License 


\section{Dysfunctional cerebrovascular tone contributes to cognitive}

impairment in a non-obese rat model of prediabetic challenge: Role of suppression of autophagy and modulation by anti-diabetic drugs

Walaa Fakih $^{1}$, Ali Mroueh ${ }^{1}$, Houssein Salah ${ }^{2}$, Ali H. Eid ${ }^{1}$, Makram Obeid ${ }^{2,3}$, Firas Kobeissy ${ }^{4}$, Hala Darwish ${ }^{5 \#}$, and Ahmed F. El-Yazbi ${ }^{1,6 \#}$

${ }^{1}$ Department of Pharmacology and Toxicology, Faculty of Medicine, American University of Beirut, Beirut, Lebanon,

${ }^{2}$ Department of Anatomy Cell Biology and Physiology, Faculty of Medicine, American University of Beirut, Beirut, Lebanon,

${ }^{3}$ Division of Child Neurology, Department of Pediatric and Adolescent Medicine, American University of Beirut Medical Center, Lebanon,

${ }^{4}$ Department of Biochemistry and Molecular Genetics, Faculty of Medicine, American University of Beirut, Beirut, Lebanon,

${ }^{5}$ Hariri School of Nursing, American University of Beirut, Beirut, Lebanon, and

${ }^{6}$ Department of Pharmacology and Toxicology, Faculty of Pharmacy, Alexandria University, Alexandria, Egypt.

\section{"Corresponding authors:}

Ahmed EL-Yazbi, BPSc, PhD, BCPS

Department of Pharmacology and Toxicology

Faculty of Medicine and Medical Centre

American University of Beirut

P.O.BOX 11-0236

Riad El-Solh 11072020

Beirut-Lebanon

Phone: +9611350 000 ext. 4779

E-mail: ae88@aub.edu.lb

Hala Darwish, PhD

Hariri School of Nursing

American University of Beirut,

Beirut, Lebanon

E-mail: hd30@aub.edu.lb 


\section{Abstract:}

Early stages of metabolic dysfunction, including prediabetes, pose a high risk for cardiovascular and cognitive impairment. While several pathological mechanisms linked advanced manifestations of metabolic deterioration, such as hypoglycemia, to neuronal inflammation and cognitive decline, little is known about the detrimental processes in effect at the early stages. To address this gap, we used a non-obese rat model of prediabetes developed in our laboratory. After 12 weeks of mild hypercaloric feeding, these rats developed hyperinsulinemia and increased fat/lean ratio without an increase in blood glucose level or body weight. These rats showed vascular dysfunction manifested as exaggerated contractility as a consequence of perivascular adipose inflammation. In this study, the mild metabolic challenge was associated with impaired hippocampal-dependent cognitive functions, spatial learning and memory and spontaneous object recognition. In line with our previous findings in this model, prediabetic rats had an augmented cerebrovascular myogenic tone demonstrated as an increased pressure-evoked contraction in pressure myography experiments on rat middle cerebral artery segments. The presumed brain hypoperfusion was accompanied by increased expression of hypoxia-inducible factor- $1 \alpha$ in the hippocampus, together with markers of mitochondrial dysfunction and increased oxidative stress. In parallel, increased p62 expression and LC3 puncta in the prediabetic rat hippocampus, as well as increased Akt and mammalian target of rapamycin phosphorylation indicated a possible repression of autophagic flux. Consequently, the examination of the hippocampal CA1 area revealed increased CD68 and IBA-1 staining consistent with microglial activation and neuroinflammation, in addition to increased TUNEL staining and caspase-3 activity indicative of elevated neuronal apoptosis. Interestingly, a two-week treatment with non-hypoglycemic doses of metformin or pioglitazone, previously shown to improve adipose inflammation and vascular function, reversed the cerebrovascular and molecular alterations in the hippocampus. This was associated with an amelioration of cognitive function. The results of the present study indicate that early metabolic challenge leads to cerebrovascular alteration potentially leading to hippocampal hypoxia and mitochondrial dysfunction. Together with suppression of autophagy, these effects culminate in hippocampal inflammation and apoptosis possibly underlying the cognitive impairment. 


\section{Keywords:}

Prediabetes, cerebral artery tone, cognitive impairment, hippocampal inflammation

\section{Abbreviations:}

MHC Mild hypercaloric diet

HIF-1 $1 \alpha \quad$ Hypoxia inducible factor- $1 \alpha$

DRP1 Dynamin-related protein 1

DHE Dihydroethidium staining

mTORC Mammalian target of rapamycin complex 


\section{Introduction:}

Type 2 diabetes represents a global pandemic with projected steep rises in incidence and prevalence over the next 20-30 years (1). This significant increase was attributed to changes in human behavior, lifestyle, and environment (2), particularly rapid dietary changes towards high saturated fat, high sugar, refined and low-fiber foods (3). With considerable health impact, the detrimental outcome of these metabolic disorders is not only restricted to increased risk of cardiovascular mortality and morbidity (4), but also accelerated cognitive decline (5). Interestingly, research findings showed that indicators of both vascular and cognitive dysfunction could coexist in the prediabetic stage preceding the diagnostic features of type 2 diabetes (6).

Prediabetes represents an early stage of metabolic impairment characterized by insulin resistance and glucose intolerance, with a similar increased risk of vascular complications (7). Significantly, recent investigation implicated this disorder in the development and progression of cognitive impairment in humans $(5,8,9)$. Several mechanisms link hyperglycemia to the development of neuronal inflammation and cognitive impairment, including increased neuronal polyol pathway flux, increased advanced glycation end-product-mediated neuronal injury, increased protein kinase C activity, and increased reactive oxygen species production, the latter activating the former pathways (10). In addition, the blood-brain barrier leakiness seems to contribute to neuronal inflammation in advanced metabolic dysfunction including diabetes and obesity (11). However, the mechanisms through which cognitive dysfunction develops during prediabebtes and insulin resistance are far less clear.

On the other hand, metabolic dysfunction is associated with a reduction of cerebral blood flow to the extent observed in patients with Alzheimer's dementia (12). Interestingly, these cerebrovascular abnormalities known to lead to cognitive decline 
are not only common in diabetes (13), but also occur in the prediabetic stage (14), where insulin resistance contributes to endothelial dysfunction and increased cerebrovascular tone (6). Indeed, a number of previous studies reported increased cerebrovascular tone in animal models of prediabetes and insulin resistance $(15,16)$ potentially contributing to chronic yet mild cerebral ischemia.

Whereas metabolic disease accelerates cognitive decline, activation of neuronal autophagy reverses the age-related memory deficits (17). Autophagy is a process of self-digestion whereby the cellular machinery recycles misfolded proteins and dysfunctional organelles, an essential activity in quiescent terminally differentiated neuronal cells (18). A considerable body of evidence implicates the reduction of autophagy in the pathogenesis of neurodegenerative disease and associated cognitive impairment (19-22). Interestingly, several studies showed that autophagy is suppressed in metabolic disorders with insulin resistance in the liver (23), and muscle (24). As well, there has been no paucity of evidence linking insulin resistance to increased protein aggregates in the brain $(25,26)$, even in the absence of hyperglycemia (27), yet the contributory role of autophagy and the effects of its potential suppression or activation remain largely unknown.

As such, we hypothesized that prediabetic metabolic dysfunction could lead to cognitive impairment via altering cerebrovascular and neuronal autophagic functions. Whereas increased cerebrovascular tone would limit blood supply and initiate hypoxic damage, suppression of autophagy potentially aggravates the insult by reducing neuronal turn-over of damaged organelles/molecules. To study this, we used a rat model of mild metabolic challenge developed in our laboratory (28-30). This rat model develops hyperinsulinemia, hyperlipidemia, and adipose inflammation in the absence of obesity, hyperglycemia, and hypertension following twelve weeks of exposure to a 
high-calorie diet. However, a gradual increase in fasting and random blood glucose levels is observed after 16 weeks of feeding, indicating the eventual incidence of diabetic hyperglycemia. As a consequence of adipose inflammation, vascular tissue from these rats demonstrated increased contractility, endothelial dysfunction, and elevated production of reactive oxygen species (28-30). Our results showed that endothelial dysfunction and increased cerebrovascular tone in this model were associated with signs of hippocampal hypoxia, mitochondrial dysfunction, suppression of autophagy, and increased hippocampal inflammation and apoptosis. This was coupled with a cognitive deficit that was reversed upon treatment with metformin or pioglitazone, as examples of anti-diabetic drugs used to treat insulin resistance. 


\section{Methods:}

\section{A. Ethical Approval}

The Institutional Animal Care and Use Committee (IACUC), in compliance with the Guide for Care and Use of Laboratory Animals of the Institute for Laboratory Animal Research of the National Academy of Sciences, USA (31), approved all animal experiments done in this paper.

\section{B. Experimental Design}

Forty Male Sprague-Dawley rats (4-5 weeks of age; $\approx 150 \mathrm{~g}$ ) were randomly divided into four groups (10 rats per group) and kept for 12 weeks: (1) rats fed with normal chow diet (control, $3 \mathrm{Kcal} / \mathrm{g}$ ), (2) rats fed with mild hypercaloric diet (MHC, 4.035 $\mathrm{Kcal} / \mathrm{g}$ ), (3) rats fed with mild hypercaloric diet and treated with $100 \mathrm{mg} / \mathrm{kg}$ metformin at week 10 (MHC-met), (4) rats fed with mild hypercaloric diet and treated with 2.5 $\mathrm{mg} / \mathrm{kg}$ pioglitazone at week 10 (MHC-pio). All rats had free access to food and water. Rats were caged individually in a temperature- and humidity-controlled room, with a 12-hour light/dark cycle. All rats were handled for 2 minutes by the experimenters and habituated to the housing, testing room, and the empty $Y$ maze used for cognitive testing for a total of 5 minutes daily, 5 consecutive days before the experiment. The treatment groups received the drugs incorporated with the MHC chow. The calculated dose was geometrically mixed with a $5 \mathrm{~g} \mathrm{HC}$ diet pellet. The drug-containing food pellet was administered once daily starting week 10 and continued for two weeks untill the end of the twelve-week feeding period. Ad libitum access to the MHC diet resumed after the drug-containing $5 \mathrm{~g}$ pellet was consumed. MHC diet was administered to the MHC-fed group in the same manner during that period. Bodyweight was determined weekly and random blood glucose levels (using ACCU-CHEK Performa glucometer, Roche Diagnostics, Rotkreuz, Switzerland) were measured using blood droplets 
collected by a tail prick. At the time of sacrifice, animals from each group were alternated between three different protocols: injection of Evans Blue dye, transcardiac perfusion and fixation, or brain collection for dissection for myography and flash freezing of dissected hippocampus in liquid nitrogen.

C. Mild hypercaloric diet preparation and composition:

MHC diet was prepared as described previously $(28,29)$. Normal chow (ENVIGO) was obtained from Teklad Rodent Diets (Madison, WI) offering $3 \mathrm{Kcal} / \mathrm{g}$ with the following contribution from macronutrients: $54 \%$ from carbohydrates, $32 \%$ from protein, and $14 \%$ from fat ( $0.9 \%$ saturated fat by weight). The MHC diet was prepared in-house and consists of food-grade fructose ( $20 \%$ by weight, Santiveri foods, Spain) and hydrogenated vegetable oil (Mazola ${ }^{\circledR}, 15 \%$ by weight, BFSA) added to the normal chow diet with the supplementation of major electrolytes as recommended by the American Institute of Nutrition (32). The final composition and calorific value were confirmed by bomb calorimetry. Macronutrient composition is as follows: by weight (calorie content) $18.06 \%$ fat $(38.68 \%, 5 \%$ saturated fat by weight), $15.8 \%$ protein $(15.66 \%)$ and $46.13 \%$ carbohydrates $(45.73 \%)$.

D. Nuclear Magnetic Resonance:

LF10 Minispec Nuclear Magnetic Resonance (NMR) machine (Bruker, MA, USA) was used to measure rat fat/lean ratio to detect different tissue densities. The values obtained from each rat are compared to a standardized, calibrated rat.

\section{E. Behavioral Testing}

Previous studies reported an early deficit in hippocampal-dependent functions upon metabolic challenge preceding these observed in functions related to other brain centers $(33,34)$. The cognitive tests used in this study, to assess hippocampaldependent function, require motor and muscle coordination, therefore we assessed 
the rats motor power using an accelerating rotarod test as described previously to rule out any deficits(21). The latency to fall, revolutions per minute of falling, and distance traveled on the rod were recorded, and the average value obtained from the four trials of the second test day was used for analysis.

1. Spontaneous object recognition using Y-Maze:

Hippocampal-dependent recognition memory was assessed using a test of spontaneous novel object recognition in a Y-maze as described previously $(35,36)$. Briefly, the rats were habituated to the empty maze for $1 \mathrm{~min}, 5$ days before the test. The test was conducted over 2 trials, 2 min each, with 15 min inter-trial delay. The rat was placed in the middle arm of the maze and allowed to explore freely. During the first trial (learning phase), two identical objects were placed equidistantly in the right and left arms of the maze ( $\left.A, A^{\prime}\right)$. During the second trial (discrimination trial), the familiar object in the left arm was replaced with a novel object (B). The maze and objects were wiped with $70 \%$ ethanol between trials to eliminate odor build-up. Novelty preference scores were calculated based on the time spent exploring the different objects as indicated previously (35). The time spent by each animal touching, sniffing, or turning around each object with head directed towards the object not more than 2 $\mathrm{cm}$ radius was considered exploration time.

2. Morris Water Maze (MWM):

Spatial learning and memory (acquisition and recall) were assessed using the MWM test as previously described $(36,37)$. A circular plastic pool $1.5 \mathrm{~m}$ in diameter) was filled with $25^{\circ} \mathrm{C}$ water. At least 3 spatial cues visible to the rats were kept constant in the room, with the experimenter standing in the same location throughout the testing period. On the first testing day, considered a habituation day, rats were allowed to swim for 2 min freely. During the spatial acquisition trials on days $2-5$, an "invisible 
platform" was placed $2 \mathrm{~cm}$ below the water surface in the Northeast Quadrant (Platform Quadrant). Rats were placed in the pool at four different equidistant immersion sites (north, east, south, and west). If a rat failed to find the platform in $2 \mathrm{~min}$, the experimenter guided and placed the animal on it for $30 \mathrm{~s}$. Immersion landmarks sequence was changed every day. On testing day 6 , the platform was removed, and the rats were immersed in water at a starting position opposite to the quadrant where the platform was located. Rats were allowed to swim freely for 2 min to assess spatial memory. On the last day, to assess motor and visual functions, rats were allowed to swim to a visible platform with 4 trials per rat. The animals were dried and kept in their home cages in the testing room between trials.

The Spontaneous object recognition test preceded the Morris Water Maze test. All behavioral tests were video recorded and analyzed with the automated SMART Video Tracking software (Panlab, Holliston, MA) with the camera suspended above the testing fields

\section{F. Assessment of blood-brain barrier leakiness}

Two percent Evans Blue dye solution in physiological saline was filtered and injected intraperitoneally at a $4 \mathrm{ml} / \mathrm{Kg}$ dose to each rat 24 hours before sacrifice (38). At sacrifice, rats were subjected to transcardiac perfusion with $50 \mathrm{ml}$ of ice-cold PBS then brain tissue was removed and divided into right and left hemispheres. The left hemisphere was weighed and snap-frozen in liquid nitrogen then stored at $-80{ }^{\circ} \mathrm{C}$. To extract and quantify the amounts of Evans Blue dye in brain tissue, the left hemisphere was homogenized in $2 \mathrm{ml}$ ice-cold PBS then sonicated for $20 \mathrm{~min}$ (30-sec on, 30-sec off). Samples were then centrifuged at $14000 \mathrm{rpm}$ for $10 \mathrm{~min}$ at $4^{\circ} \mathrm{C}$. Equal volumes of the supernatant were added to $60 \%$ trichloroacetic acid (TCA) solution. Samples were placed on a rotor for 1 hour at $4^{\circ} \mathrm{C}$ and then centrifuged at $14000 \mathrm{rpm}$ for $10 \mathrm{~min}$ at 
$4^{\circ} \mathrm{C}$. A PBS blank and a duplicate of each sample $(200 \mu \mathrm{L})$ were added to a 96 -well plate then the fluorescence was measured using $\operatorname{TriStar}^{2}$ S LB 942 Multimode Reader (Bethold, Bad Wildbad, Germany) at an excitation and emission wavelengths of 485 $\mathrm{nm}$ and $530 \mathrm{~nm}$, respectively. To serve as a positive control, the same test was conducted on a group of 4 rats that were rendered diabetic after 8 weeks of HFD feeding by a single intravenous injection of streptozotocin injection $(40 \mathrm{mg} / \mathrm{kg})$ dissolved in citrate buffer. Their diabetic status was confirmed by random blood glucose measurement showing weekly readings consistently above $300 \mathrm{mg} / \mathrm{dl}$.

\section{G. Pressure Myography}

After decapitation, the brain was immediately removed and placed in an ice-cold buffer solution containing ( $\mathrm{NaCl} 130 \mathrm{mM}, \mathrm{KCl} 4 \mathrm{mM}$, MgSO4.7H2O $1.2 \mathrm{mM}, \mathrm{NaHCO} 3$ $4 \mathrm{mM}, \mathrm{CaCl} 2.2 \mathrm{H} 2 \mathrm{O} 1.8 \mathrm{mM}$, HEPES $10 \mathrm{mM}$, KH2PO4 $1.18 \mathrm{mM}$, Glucose 6 mM, EDTA $0.03 \mathrm{mM}, \mathrm{pH}$ 7.4). Rat middle cerebral artery was isolated from surrounding connective tissue and dissected into segments of 2-3 mm of length. Cerebral artery segments were mounted in a chamber attached to a pressure myograph (DMT, Hinnerup, Denmark) for measurement of arterial diameter. Arteries were allowed to warm up to $37^{\circ} \mathrm{C}$ and equilibrate for $15-20 \mathrm{~min}$ at $10 \mathrm{~mm} \mathrm{Hg}$. The vessels were then pressurized to $60 \mathrm{mmHg}$ and allowed to develop a stable myogenic tone over $30 \mathrm{~min}$. Pressure was then dropped to $20 \mathrm{mmHg}$ and then increased again to $80 \mathrm{mmHg}$ in a series of pressure steps until a reproducible myogenic response is obtained.

In pressure ramp experiments, the pressure was dropped to $10 \mathrm{~mm} \mathrm{Hg}$ after myogenic tone development and then gradually raised in a series of steps to 10,20 , $40,60,80,100,120,140 \mathrm{~mm} \mathrm{Hg}$. The outer diameter of the vessel was measured at each pressure point. The pressure was then dropped back to $10 \mathrm{~mm} \mathrm{Hg}$ and the vessel was washed and kept in a calcium-free buffer containing $(\mathrm{NaCl} 130 \mathrm{mM}, \mathrm{KCl} 4 \mathrm{mM}$, 
MgSO4.7H2O 1.2 mM, NaHCO3 4 mM, HEPES 10 mM, KH2PO4 1.18 mM, Glucose $6 \mathrm{mM}$, EDTA $0.03 \mathrm{mM}, \mathrm{EGTA} 2 \mathrm{mM}, \mathrm{pH} \mathrm{7.4)}$ ) and the ramp was repeated. Active tone was calculated as the difference in the outer diameter of the middle cerebral artery when in calcium-containing and calcium-free buffer at the respective pressure points. To establish the active tone in denuded vessels, a small air bubble was flushed through the vessel. A lack of response to acetylcholine (ACh) was used to confirm loss of endothelium, then the experiment was performed as mentioned.

To evaluate the endothelial function, following stable myogenic response development, arteries were kept at $80 \mathrm{~mm} \mathrm{Hg}$ pressure and increasing concentrations of $A C h\left(10^{-9}-10^{-5} \mathrm{M}\right)$ were added to the bath in a semi-log manner. The outer diameter following each dose of ACh was measured and endothelium-dependent dilation was established as the difference in diameter compared to baseline diameter at $80 \mathrm{~mm}$ Hg.

\section{H. Blood Chemistry}

Blood samples were collected after decapitation and centrifuged at $5000 \mathrm{rpm}$ for 5 $\min$ at $4^{\circ} \mathrm{C}$. The supernatant serum was isolated and stored at $-80^{\circ} \mathrm{C}$ till the time of analysis. Total serum leptin, adiponectin and insulin were measured using rat leptin, rat adiponectin, and rat insulin ELISA kits (Thermo-Fisher Scientific, Waltham, MA) as per protocols supplied by the manufacturer

\section{Brain Fixation and Sectioning}

Rats were anesthetized with a ketamine/xylazine mixture (up to $80 \mathrm{mg} / \mathrm{kg}$ body weight ketamine, and $10 \mathrm{mg} / \mathrm{kg}$ body weight xylazine) after which they were subjected to transcardiac perfusion with $100 \mathrm{~mL}$ ice cold PBS, followed by $100 \mathrm{~mL} 4 \%$ paraformaldehyde. Brains were then isolated and paraffin imbedded. Later, serial sections of $8 \mu \mathrm{m}$ thickness were prepared and used for different staining techniques. 
Owing to the importance of the CA1 area of the hippocampus in spatial memory(39) and detection of novelty $(40)$ in rats, fields from this area were used for the collection of data for the different staining techniques.

\section{J. DHE Staining}

Fixed brain tissue samples were stained with $20 \mu \mathrm{M}$ dihydroethedium (Invitrogen, Carlsbad, CA) to demonstrate reactive oxygen species (ROS) load. Fluorescent images were obtained using LEICA DM6 B fluorescence imaging microscope, through the Alexa Fluor 568 filter.

\section{K. TUNEL Assay}

Terminal deoxynucleotidyl transferase dUTP nick end labeling (TUNEL) staining was performed using Click-iT Plus TUNEL Assay kit (Thermo-Fisher Scientific, Waltham, MA) following the manufacturer's instructions. Nuclei were stained with 300 nM DAPI solution in phosphate-buffered saline for 5 min. Images were taken using LEICA DM6 B fluorescent microscope (Leica Biosystems, Buffalo Grove, IL) through the Alexa Fluor 647 filter.

\section{Immunohistochemistry}

Fixed brain tissue samples were used for immunohistochemistry as described previously $(28,29)$. Briefly, hippocampal sections were incubated with either 1:500 rabbit polyclonal CD68 antibody (Abcam, Cambridge, UK) or 1:1000 recombinant antiIba1 antibody (Abcam, Cambridge, UK) overnight at $4^{\circ} \mathrm{C}$. Immunoreactivity was developed using Novolink polymer Detection Kit (Leica Biosystems, Buffalo Grove, IL) according to the manufacturer's protocol. Images were taken using OLYMPUS CX41 light microscope (Olympus, Shinjuku, Tokyo, Japan). 


\section{Immunofluorescence}

Fixed brain tissue samples were used for immunofluorescence as described previously(41). Briefly, slides were incubated overnight at $4{ }^{\circ} \mathrm{C}$ with 1:200 rabbit polyclonal anti-LC3B (Novus Biologicals, Centennial CO). The next day slides were washed and incubated with 1:100 mouse monoclonal NeuN antibody (EMD Millipore, Burlington, MA) for 2 hours at room temperature. A mixture of 1:1000 Texas redconjugated goat anti-rabbit IgG and 1:1000 Alexa Fluor 488-conjugated goat antimouse IgG (Abcam, Cambridge, UK) for 1 hour at room temperature. Nuclei were stained with $300 \mathrm{nM}$ DAPI solution in phosphate-buffered saline for $5 \mathrm{~min}$. Images were taken using LEICA DM6 B fluorescent microscope (Leica Biosystems, Buffalo Grove, IL).

N. Caspase-3 Activity Assay:

After decapitation, hippocampus tissue samples were used to quantify Caspase-3 activity using a fluorometric assay kit (Abcam, Cambridge, UK) as indicated by the supplier. The signal was quantified using $\operatorname{TriStar}^{2}$ S LB 942 Multimode Reader (Bethold, Bad Wildbad, Germany) at an excitation and emission wavelengths of 400 and $505 \mathrm{~nm}$, respectively.

O. Western Blot:

Protein extraction and Western blotting were performed as described previosly(42). Briefly, hippocampus tissue samples kept at $-80^{\circ} \mathrm{C}$ were crushed under liquid nitrogen then transferred to $400 \mu \mathrm{l}$ of a protein extraction buffer containing $1 \%$ sodium dodecylsulfate, $0.9 \% \mathrm{NaCl}, 80 \mathrm{mM}$ Tris hydrochloride (pH 6.8), and $100 \mathrm{mM}$ dithiothreitol. Samples were heated at $95^{\circ} \mathrm{C}$ for $10 \mathrm{~min}$, allowed to cool down and transferred to a rocking shaker and left overnight for protein extraction at $4^{\circ} \mathrm{C}$. Extract aliquots with equal protein content were then used for SDS-PAGE and blotting. After 
transfer and fixation, nitrocellulose membranes were blocked with $5 \%$ skimmed milk in Tris-buffered saline containing $0.1 \%$ Tween-20 for one hour at room temperature. Membranes after that were incubated in a dilution of primary antibody in $0.1 \%$ TBST (1:1000 rabbit polyclonal phospho-Akt (T308), 1:1000 rabbit polyclonal SQSTM1 / p62 antibody, 1:1000 rabbit polyclonal SIRT3, and 1:1000 rabbit polyclonal HIF-1 alpha antibody, from Abcam (Cambridge, UK); 1:1000 rabbit polyclonal Phospho-mTOR (Ser2448) antibody, 1:1000 rabbit polyclonal Phospho-DRP1 (Ser616) antibody, and 1;2000 rabbit monoclonal GAPDH, from Cell Signaling Technology (Danvers, MA) overnight at $4^{\circ} \mathrm{C}$. After washing with $0.02 \%$ TBST $(4 \times 5 \mathrm{~min})$, membranes were incubated in 1:40,000 biotin-conjugated goat anti-rabbit Ig (Abcam, Cambridge, UK) for $1 \mathrm{~h}$ at room temperature followed by washing and incubation with 1:60,000 horse radish peroxidase conjugated streptavidin (Abcam, Cambridge, UK) for 30 minutes at room temperature. Blots of the GAPDH were developed using the traditional two-step Western blotting using 1:5000 horse radish peroxidase-conjugated goat anti-rabbit secondary antibody (Abcam, Cambridge, UK) in $0.1 \%$ TBST for one hour at room temperature. After washing $2 \times 5$ min with $0.05 \%$ TBST and $2 \times 5$ min with TBS, membranes were exposed to Clarity Western ECL substrate (BioRad, Hercules, CA) for 5 min following image detection using ChemiDoc imaging system (BioRad, Hercules, CA). Band optical density was measured using Image J software, and a ratio of arbitrary density units was obtained for the protein band of interest and the density of the band representing GAPDH.

\section{P. Statistical Analysis}

Data were expressed as mean \pm standard error of the mean. Statistical significance was tested using t-test, one-way ANOVA, or two-way ANOVA followed by the appropriate post hoc test as indicated in the corresponding section in the results or 
the figure legends using GraphPad Prism software version 7. $P$ value $<0.05$ was considered statistically significant. 


\section{Results:}

\section{A. Metabolic consequences of MHC diet feeding:}

Similar to our previous results(28, 29), MHC-fed rats consumed $\sim 15 \mathrm{Kcal} /$ day more than their control counterparts (data not shown). While this did not reflect in an increased body weight for the 12-week feeding duration (Fig. 1A), an increased fat/lean ratio was observed indicative of adipose expansion (Fig. 1B). As well, a lack of increase in blood glucose level (Fig. 1C) coupled with an increased serum insulin concentration (Fig. 1D) confirmed the hyperinsulinemic prediabetic state previously reported in this model. Interestingly, despite the observed change in body composition, there was no significant alteration of serum levels of leptin (Fig. 1E) or adiponectin (Fig. 1F).

\section{B. Cerebrovascular consequences of MHC diet feeding:}

In line with our previous findings in this rat model showing increased vascular contractility (29), middle cerebral artery segments from MHC-fed rats showed an augmented myogenic response (Fig. $2 \mathrm{~A} \& 2 \mathrm{~B}$ ). The arterioles from $\mathrm{MHC}$-fed rats showed an increased active tone compared to those from control rats in the operational pressure range of the rat cerebral artery (Fig. 2C). As well, middle cerebral arterioles from $\mathrm{MHC}$ fed rats showed endothelial dysfunction manifesting as a reduction in the ACh-mediated vasodilation. Whereas vessel segments from control rats demonstrated a concentration-dependent dilation of the myogenic tone by $\mathrm{ACh}$, this response was much attenuated in segments from MHC-fed rats (Fig. 2D \& 2E). Interestingly, abolishing the vascular endothelial feedback by denuding the cerebral vessel segments equalized the active tone production in vessel segments from control and $\mathrm{MHC}$-fed rats (Fig. 2F). On the other hand, MHC feeding did not affect blood-brain 
barrier leakiness measured as the absorption of Evans blue dye in the brain. Compared to control, MHC-fed rats did not show an increased Evans blue penetration, whereas rats rendered diabetic by receiving STZ injection showed a four-fold increase (Data not shown).

\section{Cognitive and motor consequences of MHC diet feeding:}

The MHC-fed rats did not show a motor deficits on the accelerating rotarod test. Latency to fall (Control: $64.64 \pm 7.71 \mathrm{sec}$; MHC: $70.03 \pm 10.73 \mathrm{sec} ; n=10, P>0.05$ ), distance travelled on the rotarod (Control: $185.9 \pm 31.15 \mathrm{~cm}$; MHC: $203.5 \pm 38.16 \mathrm{~cm}$; $n=10, P>0.05$ ), and maximum velocity reached (Control: $13.58 \pm 0.84 \mathrm{rpm}$; MHC: $14.17 \pm 1.22 \mathrm{rpm} ; n=10, P>0.05)$ were not different between the MHC-fed and control rats. However, cognitive hippocampal-dependent functions including novel object recognition and spatial learning and memory appeared to be affected negatively by the mild metabolic dysfunction induced by twelve weeks of MHC feeding. Both control and $\mathrm{MHC}$-fed rats were able to learn the location of the rescue platform over time, demonstrated by a progressive reduction in the latency to reach platform along the successive spatial acquisition trial days of the MWM test (Fig. 3A). However, compared to controls, MHC-fed rats were slower in reaching the escape platform with significantly longer escape latencies on the third day of testing. Moreover, acquisition of place learning plateaued at a minimum on the third testing day in controls, while MHC-fed rats continued to learn at a slower pace throughout the 5 testing days. In the Probe trial, MHC-fed rats exhibited retention demonstrated by a reduced preference for the platform quadrant, spending less time in the designated area (Fig. 3B \& C). Similarly, the MHC rats showed a reduced novelty preference score indicating an impaired recognition function compared to control animals (Fig. 3D \& E). 
D. Metformin or Pioglitazone treatment reverses cerebrovascular and behavioral deficits in MHC-fed rats:

Similar to our previous findings $(28,29)$, a two-week treatment of MHC-fed rats with non-hypoglycemic doses of metformin or pioglitazone did not alter blood glucose levels (Fig. 4A), but only pioglitazone reduced serum insulin levels (Fig. 4B). As well, the cerebrovascular myogenic tone was restored to control levels (Fig. 4C), together with the vasodilatory response to ACh (Fig. 4D). These metabolic and cerebrovascular changes were associated with reversal of behavioral dysfunction as well. MHC-fed rats treated with metformin or pioglitazone demonstrated a restoration of the spatial memory with an increased preference of the platform quadrant in the Probe Trail in MWM test (Fig. 4E). Moreover, object novelty preference scores were restored to control values in MHC-fed rats receiving metformin or pioglitazone (Fig. 4F).

E. MHC feeding is associated with increased markers of hippocampal hypoxia, mitochondrial dysfunction and oxidative stress reversed by metformin or pioglitazone:

Consistent with the presumed hypoxic impact of the chronic reduction of brain perfusion due to an increased cerebrovascular tonic contraction in MHC-fed rats, HIF$1 \alpha$ expression level increased in the hippocampus (Fig. 5A). This was associated with increased markers of mitochondrial dysfunction and oxidative stress. Increased phosphorylation of dynamin-related protein 1 (DRP1) at Ser616, the site enhancing DRP1-mediated mitochondrial fission and increased ROS production, was observed in the hippocampus of MHC-fed rats (Fig. 5B). Additionally, the expression levels of the mitochondrial deacetylase, sirtuin3 (Sirt3), was reduced in the hippocampus of MHC-fed rats (Fig. 5C). Along the same lines, DHE staining showed that hippocampal 
ROS levels increased in MHC-fed rats compared to controls (Fig. 5D). Interestingly, metformin or pioglitazone treatment attenuated mitochondrial dysfunction markers and ROS staining levels in MHC-fed rats to values not significantly different from controls.

F. MHC feeding is associated with decreased hippocampal autophagy restored by metformin or pioglitazone treatment:

In order to investigate whether the hyperinsulinemic state induced by MHC feeding affected autophagy in rat hippocampus, a number of relevant markers were examined. Akt activity increased in MHC-fed rat hippocampus as indicated by elevated phosphorylation at Thr308 compared to control rats (Fig. 6A), alongside a concomitant increase in the mammalian target of rapamycin (mTORC1) phosphorylation at the Aktdependent site, Ser2448 (43) (Fig. 6B). This was accompanied by an increase in the expression level of the adaptor protein p62 in MHC-fed rats (Fig. 6C). Moreover, double immunofluorescence staining of the hippocampal neurons in the CA1 area demonstrated an increased LC3B puncta in MHC-fed rat sections (Fig. 6D). In line with its effect on metabolic, vascular, and mitochondrial dysfunction parameters, treatment with either metformin or pioglitazone ameliorated the observed increases in the Akt and mTORC1 phosphorylation, p62 expression, and LC3B puncta in MHC-fed rat hippocampus.

G. MHC feeding triggers hippocampal inflammation and apoptosis that is reversed by metformin or pioglitazone treatment:

Both CD68 and IBA1 staining showed increased microglial activation in the CA1 area consistent with increased inflammation (Fig. 7A, B \&C). Increased inflammation in this area was associated with elevated apoptotic cell death. Increased hippocampal 
neuron apoptosis in MHC-fed rats was evident as increased TUNEL staining in the hippocampal CA1 area compared to control rats (Fig. 7A \&D) and an increased caspase-3 activity in total hippocampal lysates as measured in fluorimetric activity assays (Fig. 7E). Consistent with the other functional and molecular observations, both hippocampal microglial activation and apoptosis were reduced in metformin or pioglitazone treated MHC-fed rats. 


\section{Discussion:}

Early metabolic dysfunction poses a significant health risk despite not meeting the diagnostic cut-off criteria for full-blown metabolic disorders. Prediabetes in particular, with a global prevalence estimate ranging from $35-50 \%(44)$, poses a considerable health burden owing to the associated cardiovascular and cognitive decline $(5,7)$. As such, a careful investigation of the unique mechanisms linking early metabolic challenge with cognitive decline is warranted in order to allow optimal intervention. Here, we examine the potential detrimental pathways linking prediabetic metabolic perturbation to cognitive impairment in a non-obese rat model of mild metabolic challenge. Our results suggest that cerebrovascular dysfunction leading to chronic hypoperfusion and hypoxia, coupled with suppression of neuronal autophagy culminated in neuronal inflammation and apoptotic cell death in the hippocampus.

In the present study, early metabolic challenge was induced by a mild hypercaloric diet feeding. Our rat model receives $\sim 38 \%$ of dietary calories from fat, in slight excess of the upper limit of dietary fat intake recommended by the American Diabetes Association (20-35\%) (45). Fructose was added to represent refined sugars, which together with saturated fat, are thought to be the cause of cardiovascular abnormalities in humans and animal models associated with western diets (46). Similar to our previous findings $(29,30)$, prediabetic metabolic impairment manifested as hyperinsulinemia and increased fat/lean ratio without an increase in neither blood glucose levels nor body weight after 12 weeks of $\mathrm{MHC}$ feeding enabling the examination of vascular and cognitive dysfunction at an early stage of metabolic deterioration. It is noteworthy that fasting and random hyperglycemia became detectable in this rat model by the $24^{\text {th }}$ week of exposure to $\mathrm{MHC}(29)$ indicating that the impairment observed at 12 weeks represent a bona fide prediabetic state. 
As expected, MHC-fed rats demonstrated an impaired hippocampal-dependent cognitive function. Both novelty recognition and spatial memory acquisition and recall were reduced compared to control rats. This occurred without an effect on motor function as demonstrated in the rotarod test indicating that the observed deficit is more likely to be cognitive rather than a consequence of alteration of motor activity. Significantly, adipose expansion observed here as an increased fat/lean ratio together with the perivascular adipose inflammation consistently demonstrated in this rat model $(28,29)$ might suggest that the cognitive decline develops due to an altered adipokine profile. Indeed, obesity-associated increases and decreases in circulating levels of leptin and adiponectin, respectively, are thought to mediate cognitive decline (47). However, this did not appear to be the case in our model, since no change was detected in serum levels of either adipokine in MHC-fed rats.

On the other hand, late stages of metabolic dysfunction including obesity and diabetes were shown to be associated with significant impairment of cognitive functions related to the hippocampus as a result of increased blood-brain barrier leakiness leading to neuronal inflammation (11). Yet our rat model of early metabolic challenge did not demonstrate an increased blood-brain leakiness similar to that observed in the rat group in which type 2 diabetes was induced by streptozotocin injection. This is consistent with previous studies showing that hippocampaldependent cognitive function impairment was detected in rats fed a western diet prior to an increase in blood-brain barrier leakiness and body weight (34). As such, alternative explanations for the observed cognitive impairment were sought.

Indeed, our previous observations in this prediabetic rat model showed increased vascular contractile tone and endothelial dysfunction as a consequence of perivascular adipose inflammation and mild metabolic dysfunction $(29,30)$. This was 
a consequence of an enhanced smooth muscle calcium sensitization downstream of increased basal RhoA-dependent kinase activity. An augmented vascular tone was consistently observed in other prediabetic rat models as well (15). Cerebral arterioles are among the resistance vessels producing a spontaneous myogenic contractile response in face of increasing pressure. Such a response is crucial for the maintenance of several functions including blood flow autoregulation, tissue protection, and modulation of oxygen and nutrient delivery according to regional metabolic needs (48). Our present results showed that MHC-fed rat middle cerebral arterioles produced an elevated myogenic response over the operational range of the blood vessel. Literature suggests that mean blood pressure in rat middle cerebral artery is $\sim 60 \mathrm{~mm} \mathrm{Hg} \mathrm{(49),} \mathrm{which} \mathrm{is} \mathrm{the} \mathrm{values} \mathrm{associated} \mathrm{with} \mathrm{the} \mathrm{largest} \mathrm{difference} \mathrm{in}$ active tone production between vessel segments from control and $\mathrm{MHC}$-fed rats in pressure myography experiments. Moreover, consistent with our previous findings (30), ACh-mediated endothelium-dependent relaxation of rat middle cerebral arteriole was impaired in vessel segments from MHC-fed rats. In the latter study, endothelial dysfunction was shown to be a consequence of a reduction in endothelium-dependent hyperpolarization and inward rectifier potassium channel expression. Interestingly, upon removal of the endothelial layer, denuded cerebral arteriolar segments from both control rats produced increased myogenic constriction equivalent to that generated by segments from MHC-fed rats. This indicated that augmented myogenic response observed in vessels from metabolically impaired rats is potentially due to a reduced endothelial feedback.

Owing to increased cerebral arteriole myogenic tone and reduced vessel diameter at physiological pressures, cerebral hypoperfusion and hypoxia become probable consequences in MHC-fed rats. This was confirmed by the elevated expression of 
HIF-1 $\alpha$ in MHC-fed rat hippocampus. Indeed, similar changes in HIF-1 $\alpha$ expression were seen accompanied by a decline in hippocampal-associated memory following intermittent hypoxia (50). Consistent with the literature showing that cerebral hypoxia and accumulation of HIF-1 $\alpha$ are associated with increased ROS levels(51), MHC-fed rat hippocampus demonstrated a high oxidative burden upon DHE staining. Moreover, mitochondrial dysfunction is a common occurrence in hypoxic neuronal injury (52). As such, we proceeded to examine two mitochondrial markers; DRP1 phosphorylation at Ser616 promoting mitochondrial fission (53), and Sirt3 that plays a role in maintaining mitochondrial antioxidant function and biogenesis (54). Studies have shown that hypoxia triggered a feedback mechanism downregulating mitochondrial activity through increased fragmentation, mitophagy, and reduced biogenesis $(55,56)$. Specifically, recent studies reported that hypoxia-induced mitochondrial fission via increased HIF-1 $\alpha$ expression leading to increased DRP1 activity (57-59). This was indeed the case in the hippocampus of MHC-fed rats where increased DRP1 phosphorylation at Ser616 was observed. Furthermore, as previously observed in HFD-fed mice showing cognitive dysfunction (60), Sirt3 expression levels were reduced in MHC-fed rat hippocampus.

In the context of neuronal injury, evidence showed that cells respond to increased mitochondrial fission by triggering autophagy $(56,61)$ as a quality control mechanism to clear damaged mitochondrial segments $(62,63)$. Indeed, autophagy activation was reported to have a neuroprotective role, not only in neurodegenerative disease (21, $64)$, but also under hypoxic conditions $(65,66)$. Yet, this did not appear to be the case in MHC-fed rats. In line with the early metabolic challenge manifesting as hyperinsulinemia, the hippocampus of MHC-fed rats showed increased Akt phosphorylation at Thr308. This in turn was associated with an increased mTORC 
phosphorylation at the Akt sensitive site, Ser2448 (43). Multiple lines of evidence implicate the activation of the insulin receptor/Akt/mTORC signaling pathway in suppression of autophagy associated with insulin resistance in neurons (67) and peripheral tissue (68). mTORC activation represses the activity of the unc-51-like kinase 1 (ULK1) via phosphorylation, and thus precludes autophagosome maturation (69). Additionally, recent findings revealed an additional mechanism whereby ULK1 inhibition contributes to autophagy suppression through prevention of autophagosome and lysosome fusion (70). In this context, MHC-fed rat hippocampus showed accumulation of the adaptor protein SQSTM1/p62 and increased LC3 puncta in neuronal cells in the CA1 area indicative of an impeded autophagy flux. Interestingly, studies of CA1 neurons of Alzheimer's patients showed a similar picture (71).

In parallel, examination of the hippocampus in rats with early metabolic challenge revealed an increase in markers of inflammation and apoptosis. On the one hand, MHC-fed rats showed increased expression of CD68 and IBA-1, both markers of microglial activation associated with chronic neuroinflammation (72), in the CA1 area of the hippocampus. On the other hand, increased apoptotic changes in MHC-fed rat hippocampus appeared as increased TUNEL staining and higher caspase-3 activity. Similar observations are common in recent literature whereby neuroinflammation and microglial activation (73-75), together with hippocampal apoptosis $(76,77)$, occur in association with high fat diet-induced cognitive decline. The previous studies provided observational evidence for the aforementioned association. However, in the context of the current study, it is possible that the impeded autophagic flux in MHC-fed rat hippocampus was the trigger for activation of apoptosis and inflammation as previously observed in neuronal and non-neuronal tissues (78-80). 
From a different perspective, our previous work showed that the amelioration of adipose inflammation in this rat model of early metabolic challenge was associated with reversal of the vascular dysfunction in vitro and in vivo $(28,29)$. In the present study, the same non-hypoglycemic doses of metformin and pioglitazone used to ameliorate adipose inflammation in the previous work led to improved cerebrovascular function. This was also associated with an improved performance in MWM and Ymaze tests. Similar to previous results(29), the only metabolic impact was observed in the pioglitazone-treated group as a reduction of serum insulin level, consistent with its insulin-sensitizing effect. Despite the lack of a detectable change in HIF-1 $\alpha$ expression levels, improvement of cerebrovascular function in the treated groups was associated with favorable changes in the mitochondrial markers, DRP1 phosphorylation and Sirt3 expression, as well as with reduced oxidative stress. While presumed to be related to brain perfusion, an absence of a detectable decrease in HIF-1 $\alpha$ expression level is not unexpected given that prior studies on diabetic rat models showed that significant changes were only observed after at least one month of intervention (81). Nevertheless, treatment by metformin and pioglitazone reversed the molecular changes in the pathways leading to suppression of autophagy. While a reduction in Akt phosphorylation, and the subsequent mTORC activation, are consistent with reduced serum insulin levels in the pioglitazone-treated MHC-fed rat group, prior evidence reported a direct inhibitory effect for metformin on Akt, as well as mTORC, phosphorylation and activation (82). This was associated with a reversal of the changes seen in the autophagy markers p62 and LC3. Along the same lines, the CA1 area of the hippocampus in metformin- and pioglitazone-treated MHC rats showed reduced markers of microglial activation and apoptosis. 
In conclusion, our present results propose a possible framework for a continuum of events linking peripheral inflammatory changes in early metabolic dysfunction to cognitive decline. Cerebrovascular changes, occurring in the context of wider vascular dysfunction secondary to metabolic alteration, lead to hippocampal hypoxia with possible mitochondrial dysfunction and increased oxidative stress. In parallel, the altered metabolic profile potentially contributes to a reduced autophagic flux precluding the neuronal rescue mechanism and thus leads to the activation of apoptosis and neuroinflammation observed alongside cognitive impairment in this model. Amelioration of the peripheral inflammatory process improves vascular, molecular, and behavioral alterations highlighting both the feasibility and importance of intervention to reverse the cognitive impact of this early stage of metabolic dysfunction. 


\section{Declarations:}

Ethics approval and consent to participate: Animal experiments in this study were conducted according to a study protocol approved by the Institutional Animal Care and Use Committee at the American University of Beirut and in accordance with the Guide for Care and Use of Laboratory Animals of the Institute for Laboratory Animal Research of the National Academy of Sciences, USA.

Consent for publication: Not applicable.

Availability of data and materials: All data generated or analyzed during this study are included in this published article.

Competing interests: None to declare.

Funding: This study was supported by a joint grant funded by the National Council for Scientific Research in Lebanon (CNRS-L) and the American University of Beirut $(A \cup B)$. The funding bodies had no role in the design of the study or collection, analysis, and interpretation of data or in writing the manuscript.

Authors' contributions: AFE was responsible for the conception and design of experiments. WF performed behavioral and molecular experiments with assistance from HS. AM performed pressure myography experiments. AHE, MO, HD, FK and AFE collected, analyzed, and interpreted data. AFE and WF wrote the manuscript. AFE supervised and supported the study. All authors read the journal authorship agreement, read the manuscript, provided feedback, and approved the manuscript final form.

Acknowledgements: Not applicable

Author information: Current Address for Walaa Fakih is INSERM UMR1260 Regenerative NanoMedicine, Fédération de Médecine Translationnelle de Strasbourg, Université de Strasbourg, Faculté de Pharmacie, 67401 Illkirch, France. 


\section{References:}

1. International_Diabetes_Federation. IDF Diabetes Atlas, 8 ed. Brussels, Belgium: International Diabetes Federation2017.

2. Kuhnlein HV, Receveur O. Dietary change and traditional food systems of indigenous peoples. Annu Rev Nutr. 1996;16:417-42.

3. Misra A, Singhal N, Khurana L. Obesity, the metabolic syndrome, and type 2 diabetes in developing countries: role of dietary fats and oils. Journal of the American College of Nutrition. 2010;29(3 Suppl):289s-301s.

4. Leon BM, Maddox TM. Diabetes and cardiovascular disease: Epidemiology, biological mechanisms, treatment recommendations and future research. World journal of diabetes. 2015;6(13):1246-58.

5. Marseglia A, Fratiglioni L, Kalpouzos G, Wang R, Backman L, Xu W. Prediabetes and diabetes accelerate cognitive decline and predict microvascular lesions: A population-based cohort study. Alzheimer's \& dementia : the journal of the Alzheimer's Association. 2019;15(1):25-33.

6. Hughes TM, Craft S. The role of insulin in the vascular contributions to age-related dementia. Biochimica et Biophysica Acta (BBA) - Molecular Basis of Disease. 2016;1862(5):983-91.

7. Tabak AG, Herder C, Rathmann W, Brunner EJ, Kivimaki M. Prediabetes: a high-risk state for diabetes development. Lancet (London, England). 2012;379(9833):2279-90.

8. Dybjer E, Nilsson PM, Engström G, Helmer C, Nägga K. Pre-diabetes and diabetes are independently associated with adverse cognitive test results: a cross-sectional, populationbased study. BMC Endocrine Disorders. 2018;18(1):91.

9. Lutski M, Weinstein G, Goldbourt U, Tanne D. Insulin Resistance and Future Cognitive Performance and Cognitive Decline in Elderly Patients with Cardiovascular Disease. Journal of Alzheimer's disease : JAD. 2017;57(2):633-43.

10. Kodl CT, Seaquist ER. Cognitive dysfunction and diabetes mellitus. Endocr Rev. 2008;29(4):494-511.

11. Van Dyken P, Lacoste B. Impact of Metabolic Syndrome on Neuroinflammation and the Blood-Brain Barrier. Front Neurosci. 2018;12:930-.

12. Biessels GJ, Kappelle AC, Bravenboer B, Erkelens DW, Gispen WH. Cerebral function in diabetes mellitus. Diabetologia. 1994;37(7):643-50.

13. Ergul A, Kelly-Cobbs A, Abdalla M, Fagan SC. Cerebrovascular complications of diabetes: focus on stroke. Endocrine, metabolic \& immune disorders drug targets. 2012;12(2):148-58.

14. Rusinek H, Ha J, Yau PL, Storey P, Tirsi A, Tsui WH, et al. Cerebral Perfusion in Insulin Resistance and Type 2 Diabetes. Journal of Cerebral Blood Flow \& Metabolism. 2015;35(1):95-102.

15. Abd-Elrahman KS, Colinas O, Walsh EJ, Zhu HL, Campbell CM, Walsh MP, et al. Abnormal myosin phosphatase targeting subunit 1 phosphorylation and actin polymerization contribute to impaired myogenic regulation of cerebral arterial diameter in the type 2 diabetic Goto-Kakizaki rat. Journal of cerebral blood flow and metabolism : official journal of the International Society of Cerebral Blood Flow and Metabolism. 2017;37(1):227-40.

16. Butcher JT, Goodwill AG, Stanley SC, Frisbee JC. Differential impact of dilator stimuli on increased myogenic activation of cerebral and skeletal muscle resistance arterioles in obese zucker rats. Microcirculation (New York, NY : 1994). 2013;20(7):579-89.

17. Glatigny M, Moriceau S, Rivagorda M, Ramos-Brossier M, Nascimbeni AC, Lante F, et al. Autophagy Is Required for Memory Formation and Reverses Age-Related Memory Decline. Current biology : CB. 2019;29(3):435-48.e8. 
18. Son JH, Shim JH, Kim K-H, Ha J-Y, Han JY. Neuronal autophagy and neurodegenerative diseases. Experimental \&Amp; Molecular Medicine. 2012;44:89.

19. Tramutola A, Triplett JC, Di Domenico F, Niedowicz DM, Murphy MP, Coccia R, et al. Alteration of mTOR signaling occurs early in the progression of Alzheimer disease (AD): analysis of brain from subjects with pre-clinical $\mathrm{AD}$, amnestic mild cognitive impairment and late-stage AD. Journal of neurochemistry. 2015;133(5):739-49.

20. Kim YD, Jeong EI, Nah J, Yoo S-M, Lee WJ, Kim Y, et al. Pimozide reduces toxic forms of tau in TauC3 mice via 5' adenosine monophosphate-activated protein kinase-mediated autophagy. Journal of neurochemistry. 2017;142(5):734-46.

21. Abd-Elrahman KS, Hamilton A, Hutchinson SR, Liu F, Russell RC, Ferguson SSG. mGluR5 antagonism increases autophagy and prevents disease progression in the zQ175 mouse model of Huntington's disease. Science signaling. 2017;10(510).

22. Hara T, Nakamura K, Matsui M, Yamamoto A, Nakahara Y, Suzuki-Migishima R, et al. Suppression of basal autophagy in neural cells causes neurodegenerative disease in mice. Nature. 2006;441(7095):885-9.

23. Liu HY, Han J, Cao SY, Hong T, Zhuo D, Shi J, et al. Hepatic autophagy is suppressed in the presence of insulin resistance and hyperinsulinemia: inhibition of FoxO1-dependent expression of key autophagy genes by insulin. The Journal of biological chemistry. 2009;284(45):31484-92.

24. He C, Bassik MC, Moresi V, Sun K, Wei Y, Zou Z, et al. Exercise-induced BCL2regulated autophagy is required for muscle glucose homeostasis. Nature. 2012;481(7382):5115 .

25. Vandal M, White PJ, Tremblay C, St-Amour I, Chevrier G, Emond V, et al. Insulin reverses the high-fat diet-induced increase in brain Abeta and improves memory in an animal model of Alzheimer disease. Diabetes. 2014;63(12):4291-301.

26. Busquets O, Ettcheto M, Pallas M, Beas-Zarate C, Verdaguer E, Auladell C, et al. Long-term exposition to a high fat diet favors the appearance of beta-amyloid depositions in the brain of C57BL/6J mice. A potential model of sporadic Alzheimer's disease. Mechanisms of ageing and development. 2017;162:38-45.

27. Takalo M, Haapasalo A, Martiskainen H, Kurkinen KM, Koivisto H, Miettinen P, et al. High-fat diet increases tau expression in the brain of T2DM and AD mice independently of peripheral metabolic status. The Journal of nutritional biochemistry. 2014;25(6):634-41.

28. Al-Assi O, Ghali R, Mroueh A, Kaplan A, Mougharbil N, Eid AH, et al. Cardiac Autonomic Neuropathy as a Result of Mild Hypercaloric Challenge in Absence of Signs of Diabetes: Modulation by Antidiabetic Drugs. Oxid Med Cell Longev. 2018;2018:9389784.

29. Elkhatib MAW, Mroueh A, Rafeh RW, Sleiman F, Fouad H, Saad EI, et al. Amelioration of perivascular adipose inflammation reverses vascular dysfunction in a model of nonobese prediabetic metabolic challenge: potential role of antidiabetic drugs. Translational research : the journal of laboratory and clinical medicine. 2019.

30. Alaaeddine R, El-Khatib M, Mroueh A, Fouad H, Saad E, El-Sabban M, et al. Impaired endothelium-dependent hyperpolarization underlies endothelial dysfunction during early metabolic challenge: Increased ROS generation and possible interference with NO function. Journal of Pharmacology and Experimental Therapeutics. 2019:jpet.119.262048.

31. National Research Council Committee for the Update of the Guide for the C, Use of Laboratory A. The National Academies Collection: Reports funded by National Institutes of Health. In: th, editor. Guide for the Care and Use of Laboratory Animals. Washington (DC): National Academies Press (US)

National Academy of Sciences.; 2011. 
32. Reeves PG, Nielsen FH, Fahey GC, Jr. AIN-93 purified diets for laboratory rodents: final report of the American Institute of Nutrition ad hoc writing committee on the reformulation of the AIN-76A rodent diet. The Journal of nutrition. 1993;123(11):1939-51.

33. Davidson TL, Monnot A, Neal AU, Martin AA, Horton JJ, Zheng W. The effects of a high-energy diet on hippocampal-dependent discrimination performance and blood-brain barrier integrity differ for diet-induced obese and diet-resistant rats. Physiology \& behavior. 2012;107(1):26-33.

34. Davidson TL, Hargrave SL, Swithers SE, Sample CH, Fu X, Kinzig KP, et al. Interrelationships among diet, obesity and hippocampal-dependent cognitive function. Neuroscience. 2013;253:110-22.

35. Miedel CJ, Patton JM, Miedel AN, Miedel ES, Levenson JM. Assessment of Spontaneous Alternation, Novel Object Recognition and Limb Clasping in Transgenic Mouse Models of Amyloid-beta and Tau Neuropathology. Journal of visualized experiments : JoVE. 2017(123).

36. Briones TL, Darwish H. Vitamin D mitigates age-related cognitive decline through the modulation of pro-inflammatory state and decrease in amyloid burden. Journal of neuroinflammation. 2012;9:244.

37. Medlej Y, Salah H, Wadi L, Saad S, Bashir B, Allam J, et al. Lestaurtinib (CEP-701) modulates the effects of early life hypoxic seizures on cognitive and emotional behaviors in immature rats. Epilepsy \& Behavior. 2019;92:332-40.

38. Manaenko A, Chen H, Kammer J, Zhang JH, Tang J. Comparison Evans Blue injection routes: Intravenous versus intraperitoneal, for measurement of blood-brain barrier in a mice hemorrhage model. J Neurosci Methods. 2011;195(2):206-10.

39. Ocampo AC, Squire LR, Clark RE. Hippocampal area CA1 and remote memory in rats. Learning \& memory (Cold Spring Harbor, NY). 2017;24(11):563-8.

40. Larkin MC, Lykken C, Tye LD, Wickelgren JG, Frank LM. Hippocampal output area CA1 broadcasts a generalized novelty signal during an object-place recognition task. Hippocampus. 2014;24(7):773-83.

41. El-Yazbi AF, Cho WJ, Schulz R, Daniel EE. Caveolin-1 knockout alters betaadrenoceptors function in mouse small intestine. American journal of physiology Gastrointestinal and liver physiology. 2006;291(6):G1020-30.

42. El-Yazbi AF, Ibrahim KS, El-Gowelli HM, El-Deeb NM, El-Mas MM. Modulation by NADPH oxidase of the chronic cardiovascular and autonomic interaction between cyclosporine and NSAIDs in female rats. European journal of pharmacology. 2017;806:96104.

43. Memmott RM, Dennis PA. Akt-dependent and -independent mechanisms of mTOR regulation in cancer. Cellular signalling. 2009;21(5):656-64.

44. Hostalek U. Global epidemiology of prediabetes - present and future perspectives. Clinical diabetes and endocrinology. 2019;5:5.

45. Evert AB, Boucher JL, Cypress M, Dunbar SA, Franz MJ, Mayer-Davis EJ, et al. Nutrition therapy recommendations for the management of adults with diabetes. Diabetes Care. 2014;37 Suppl 1:S120-43.

46. Valensi P. Hypertension, single sugars and fatty acids. J Hum Hypertens. 2005;19 Suppl 3:S5-9.

47. Forny-Germano L, De Felice FG, Vieira M. The Role of Leptin and Adiponectin in Obesity-Associated Cognitive Decline and Alzheimer's Disease. Front Neurosci. 2018;12:1027.

48. El-Yazbi AF, Abd-Elrahman KS. ROK and Arteriolar Myogenic Tone Generation: Molecular Evidence in Health and Disease. Frontiers in pharmacology. 2017;8:87. 
49. Heistad DD. What's new in the cerebral microcirculation? Landis Award lecture. Microcirculation (New York, NY : 1994). 2001;8(6):365-75.

50. Arias-Cavieres A, Khuu MA, Nwakudu CU, Barnard JE, Dalgin G, Garcia AJ. A role for Hypoxia Inducible Factor 1a (HIF1a) in intermittent hypoxia-dependent changes to spatial memory and synaptic plasticity. bioRxiv. 2019:595975.

51. Chen R, Lai UH, Zhu L, Singh A, Ahmed M, Forsyth NR. Reactive Oxygen Species Formation in the Brain at Different Oxygen Levels: The Role of Hypoxia Inducible Factors. Front Cell Dev Biol. 2018;6:132.

52. Zhao XY, Lu MH, Yuan DJ, Xu DE, Yao PP, Ji WL, et al. Mitochondrial Dysfunction in Neural Injury. Front Neurosci. 2019;13:30.

53. Chang CR, Blackstone C. Dynamic regulation of mitochondrial fission through modification of the dynamin-related protein Drp1. Annals of the New York Academy of Sciences. 2010;1201:34-9.

54. Sidorova-Darmos E, Sommer R, Eubanks JH. The Role of SIRT3 in the Brain Under Physiological and Pathological Conditions. Frontiers in Cellular Neuroscience. 2018;12(196).

55. Ježek J, Cooper KF, Strich R. Reactive Oxygen Species and Mitochondrial Dynamics: The Yin and Yang of Mitochondrial Dysfunction and Cancer Progression. Antioxidants (Basel, Switzerland). 2018;7(1):13.

56. Thomas LW, Ashcroft M. Exploring the molecular interface between hypoxiainducible factor signalling and mitochondria. Cell Mol Life Sci. 2019;76(9):1759-77.

57. Zhang D, Liu Y, Tang Y, Wang X, Li Z, Li R, et al. Increased mitochondrial fission is critical for hypoxia-induced pancreatic beta cell death. PloS one. 2018;13(5):e0197266.

58. Kuo CW, Tsai MH, Lin TK, Tiao MM, Wang PW, Chuang JH, et al. mtDNA as a Mediator for Expression of Hypoxia-Inducible Factor 1alpha and ROS in Hypoxic Neuroblastoma Cells. Int J Mol Sci. 2017;18(6).

59. Kim DY, Jung SY, Kim YJ, Kang S, Park JH, Ji ST, et al. Hypoxia-dependent mitochondrial fission regulates endothelial progenitor cell migration, invasion, and tube formation. The Korean Journal of Physiology \& Pharmacology : Official Journal of the Korean Physiological Society and the Korean Society of Pharmacology. 2018;22(2):203-13.

60. Shi Z, Li C, Yin Y, Yang Z, Xue H, Mu N, et al. Aerobic Interval Training Regulated SIRT3 Attenuates High-Fat-Diet-Associated Cognitive Dysfunction. BioMed research international. 2018;2018:2708491-.

61. Balog J, Mehta SL, Vemuganti R. Mitochondrial fission and fusion in secondary brain damage after CNS insults. Journal of cerebral blood flow and metabolism : official journal of the International Society of Cerebral Blood Flow and Metabolism. 2016;36(12):2022-33.

62. Twig G, Elorza A, Molina AJ, Mohamed H, Wikstrom JD, Walzer G, et al. Fission and selective fusion govern mitochondrial segregation and elimination by autophagy. Embo $\mathrm{j}$. 2008;27(2):433-46.

63. Chen H, Chan DC. Mitochondrial dynamics--fusion, fission, movement, and mitophagy--in neurodegenerative diseases. Hum Mol Genet. 2009;18(R2):R169-R76.

64. Puyal J, Ginet V, Grishchuk Y, Truttmann AC, Clarke PGH. Neuronal Autophagy as a Mediator of Life and Death:Contrasting Roles in Chronic Neurodegenerative and Acute Neural Disorders. The Neuroscientist. 2012;18(3):224-36.

65. Shi ZY, Deng JX, Fu S, Wang L, Wang Q, Liu B, et al. Protective effect of autophagy in neural ischemia and hypoxia: Negative regulation of the Wnt/beta-catenin pathway. International journal of molecular medicine. 2017;40(6):1699-708.

66. Balduini W, Carloni S, Buonocore G. Autophagy in hypoxia-ischemia induced brain injury. The Journal of Maternal-Fetal \& Neonatal Medicine. 2012;25(sup1):30-4.

67. de Mello NP, Orellana AM, Mazucanti CH, de Morais Lima G, Scavone C, Kawamoto EM. Insulin and Autophagy in Neurodegeneration. Front Neurosci. 2019;13(491). 
68. Ehrlicher SE, Stierwalt HD, Newsom SA, Robinson MM. Skeletal muscle autophagy remains responsive to hyperinsulinemia and hyperglycemia at higher plasma insulin concentrations in insulin-resistant mice. Physiological reports. 2018;6(14):e13810.

69. Paquette M, El-Houjeiri L, Pause A. mTOR Pathways in Cancer and Autophagy. Cancers. 2018;10(1).

70. Wang C, Wang H, Zhang D, Luo W, Liu R, Xu D, et al. Phosphorylation of ULK1 affects autophagosome fusion and links chaperone-mediated autophagy to macroautophagy. Nature communications. 2018;9(1):3492.

71. Bordi M, Berg MJ, Mohan PS, Peterhoff CM, Alldred MJ, Che S, et al. Autophagy flux in CA1 neurons of Alzheimer hippocampus: Increased induction overburdens failing lysosomes to propel neuritic dystrophy. Autophagy. 2016;12(12):2467-83.

72. Hendrickx DAE, van Eden CG, Schuurman KG, Hamann J, Huitinga I. Staining of HLA-DR, Iba1 and CD68 in human microglia reveals partially overlapping expression depending on cellular morphology and pathology. Journal of neuroimmunology. 2017;309:1222.

73. Duffy CM, Hofmeister JJ, Nixon JP, Butterick TA. High fat diet increases cognitive decline and neuroinflammation in a model of orexin loss. Neurobiology of learning and memory. 2019;157:41-7.

74. Spencer SJ, D'Angelo H, Soch A, Watkins LR, Maier SF, Barrientos RM. High-fat diet and aging interact to produce neuroinflammation and impair hippocampal- and amygdalardependent memory. Neurobiology of aging. 2017;58:88-101.

75. Duan Y, Zeng L, Zheng C, Song B, Li F, Kong X, et al. Inflammatory Links Between High Fat Diets and Diseases. Frontiers in immunology. 2018;9(2649).

76. Nakandakari S, Munoz VR, Kuga GK, Gaspar RC, Sant'Ana MR, Pavan ICB, et al. Short-term high-fat diet modulates several inflammatory, ER stress, and apoptosis markers in the hippocampus of young mice. Brain Behav Immun. 2019;79:284-93.

77. Qin S, Sun D, Zhang C, Tang Y, Zhou F, Zheng K, et al. Downregulation of sonic hedgehog signaling in the hippocampus leads to neuronal apoptosis in high-fat diet-fed mice. Behavioural brain research. 2019;367:91-100.

78. Mariño G, Niso-Santano M, Baehrecke EH, Kroemer G. Self-consumption: the interplay of autophagy and apoptosis. Nature reviews Molecular cell biology. 2014;15(2):8194.

79. Wei H, Yin M, Lu Y, Yang Y, Li B, Liao X-X, et al. Mild hypothermia improves neurological outcome in mice after cardiopulmonary resuscitation through Silent Information Regulator 1-actviated autophagy. Cell Death Discov. 2019;5:129-.

80. Chen J, Wang L, Wu C, Hu Q, Gu C, Yan F, et al. Melatonin-enhanced autophagy protects against neural apoptosis via a mitochondrial pathway in early brain injury following a subarachnoid hemorrhage. Journal of Pineal Research. 2014;56(1):12-9.

81. Gu L, Xu H, Zhang C, Yang Q, Zhang L, Zhang J. Time-dependent changes in hypoxiaand gliosis-related factors in experimental diabetic retinopathy. Eye (Lond). 2019;33(4):6009.

82. Würth R, Pattarozzi A, Gatti M, Bajetto A, Corsaro A, Parodi A, et al. Metformin selectively affects human glioblastoma tumor-initiating cell viability. Cell Cycle. 2013;12(1):145-56. 


\section{Figure Legends:}

Figure 1. Metabolic consequences of 12 weeks of MHC-feeding. Time course of change in body weight $(A)$, body composition represented as fat/lean ratio $(B)$, random blood glucose level (C), serum insulin levels (D), serum leptin (E), and serum adiponectin concentrations $(F)$ after 12 weeks of feeding in control and MHC-fed rats. Data summarized represent results from ten rats per group. For $A$, statistical analysis was done by two-way ANOVA followed by Sidak's multiple comparisons test, while for B-F unpaired $t$-test was used. * denotes $P<0.05$ vs. control rats.

Figure 2. Cerebrovascular tone and endothelial function in control and metabolically challenged rats. $A$, Representative tracings of pressure myography experiments depicting changes in rat middle cerebral artery diameter as a function of change in intravascular pressure in vessel segments from control (left) and MHC-fed (right) rats in presence $(+\mathrm{Ca})$ and absence $(-\mathrm{Ca})$ of calcium in the bath solution; $\mathrm{B}$, summary of the change in middle cerebral artery diameter as a function of pressure change; C, Active tone generated by rat middle cerebral artery in vessel segments from control and MHC-fed rats. Active tone is the difference in diameter of the vessel at a given intravascular pressure value in absence and presence of calcium; $D$, Representative tracings of the endothelium-dependent vasodilatory effects of increasing ACh concentrations in rat middle cerebral artery segments producing stable myogenic tone during pressurization to $80 \mathrm{~mm} \mathrm{Hg}$ from control (left) and MHC-fed rats (right); E, Increase in rat middle cerebral artery diameter in response to increasing ACh concentrations; F, Active tone generated by rat middle cerebral artery segments after endothelium denudation. Data summarized represent results from vessel segments from four different rats per group. Statistical analysis was done by two-way ANOVA followed by Sidak's multiple comparisons test. * denotes $P<0.05$ vs. the corresponding value in control rats.

Figure 3. Early metabolic challenge is associated with impaired hippocampaldependent cognitive functions. $A$, Latency to platform on in different training days in MWM test in control and MHC-fed rats; $\mathrm{B}$, Representative tracings of rat activity in MWM probe trial; C, Time spent by control or MHC-fed rats in different quadrants in the probe trial of MWM test; D, Representative tracings of rat activity in Y-maze test; E, Novelty preference score for control and MHC-fed rats in novel object recognition test in the $\mathrm{Y}$-maze. Data summarized represent results from ten rats per group. For $A$ \& $C$, statistical analysis was done by two-way ANOVA followed by Sidak's multiple 
comparisons test, while for $\mathrm{E}$ unpaired $t$-test was used. * denotes $P<0.05$ vs. the corresponding value in control rats, while \# denotes $P<0.05$ vs. the latency value on the first training session or the control value in the NE quadrant for $A$ and $C$, respectively.

Figure 4. Metformin and pioglitazone treatment reversed cerebrovascular and cognitive deficits in MHC-fed rats. Random blood glucose level (A), serum insulin level (B), active myogenic tone of rat middle cerebral artery (C), ACh-mediated endothelium-dependent dilation of middle cerebral artery (D), time spent in different quadrants in MWM (E), and novelty preference score in novel object recognition in $Y$ maze test $(F)$ in MHC-fed rats with or without a two-week oral treatment with nonhypoglycemic doses of metformin or pioglitazone. Data summarized represent results from ten rats per group for $A, B, E$, and $F$ and four rats per group for $C$ and $D$. For $A$, $B$, and $F$ statistical analysis was done by one way ANOVA followed by Tukey multiple comparisons test, while for C, D, and E two-way ANOVA followed by Sidak's multiple comparisons test was used. ${ }^{*}$ denotes $P<0.05$ vs. the corresponding value in control rats.

Figure 5. Increased markers of hypoxia, mitochondrial dysfunction, and oxidative stress in metabolically challenged rat hippocampus recovered after treatment. HIF-1 $\alpha$ expression (A), Ser616 DRP1 phosphorylation (B), and Sirt3 expression (C) levels, together with DHE staining (D) in hippocampus from control, MHC-fed, and MHC-fed rats treated for two weeks by either metformin or pioglitazone. Western blots shown are representative of four separate experiments on extracts from four different rats per group. DHE staining appears as red fluorescence on the representative micrographs. Scale bare are $50 \mu \mathrm{m}$. DHE data are a summary of nine sections from three different rats per group. Statistical analysis was done by one way ANOVA followed by Tukey multiple comparisons test. * denotes $P<0.05$ vs. the corresponding value in control rats.

Figure 6. Activation of the Akt/mTORC pathway and decreased autophagy in hippocampus in metabolically challenged rats reversed by treatment. Akt phosphorylation at Thr308 (A), mTORC phosphorylation at Ser2448 (B), and p62 expression levels $(\mathrm{C})$, together with LC3 puncta in hippocampus from control, MHCfed, and MHC-fed rats treated for two weeks by either metformin or pioglitazone. Western blots shown are representative of four separate experiments on extracts from 
four different rats per group. NeuN staining appears as green fluorescence, LC3 staining appears as red fluorescence, while DAPI nuclear staining appear as blue fluorescence on the representative micrographs. Cells expressing both NeuN and LC3 show yellow fluorescence in the merged image. Scale bare are $5 \mu \mathrm{m}$. Immunofluorescence data are a summary of nine sections from three different rats per group. Statistical analysis was done by one way ANOVA followed by Tukey multiple comparisons test. * denotes $P<0.05$ vs. the corresponding value in control rats.

Figure 7. Increased microglial activation and apoptosis in hippocampus in metabolically challenged rats reversed by treatment. $A$, Representative micrographs of CD68, IBA-1, and TUNEL staining in CA1 area of rat hippocampus with the corresponding quantification in $\mathrm{B}, \mathrm{C} \& \mathrm{D}$, respectively. CD68 and IBA-1 staining appear as brown color on a background of H\&E staining, while TUNEL staining appear as red fluorescence together with the blue fluorescene of DAPI nuclear staining on the representative micrographs. Scale bare are $25 \mu \mathrm{m}$. Microscopy data are a summary of nine sections from three different rats per group. D, Caspase-3 activity normalized to the hippocampal extract protein concentration. Statistical analysis was done by one way ANOVA followed by Tukey multiple comparisons test. * denotes $P<0.05$ vs. the corresponding value in control rats. 
Figure 1

A

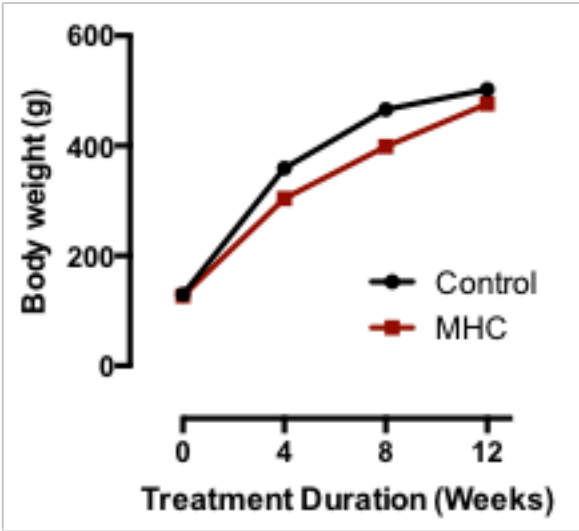

D

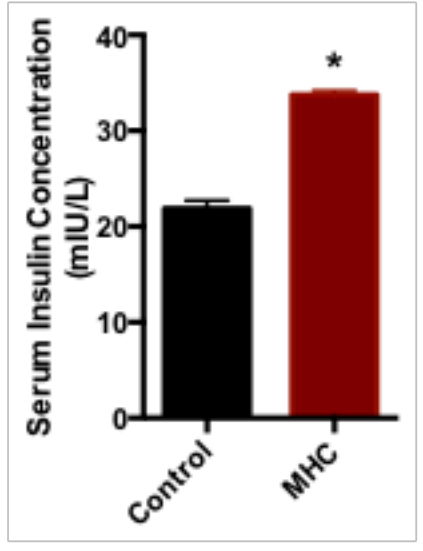

B

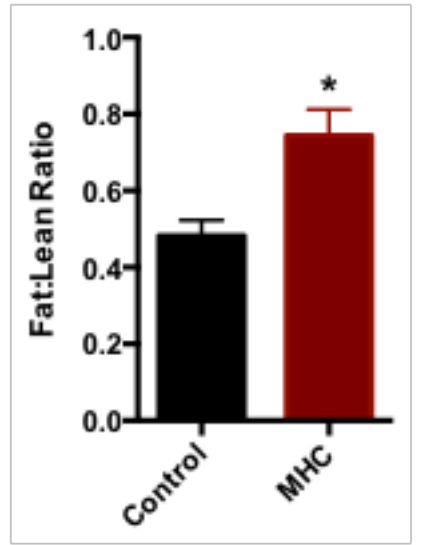

E

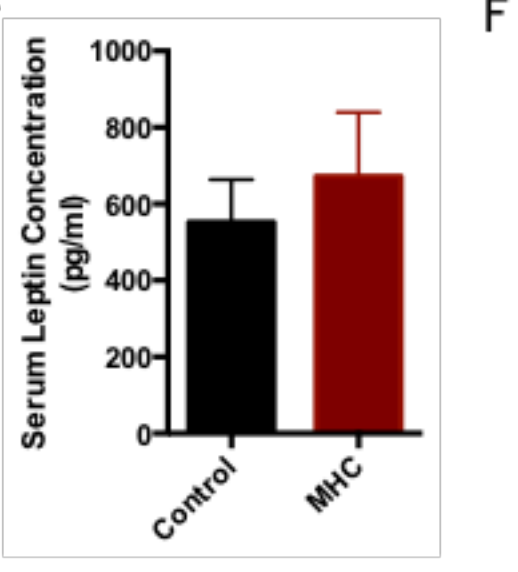

C
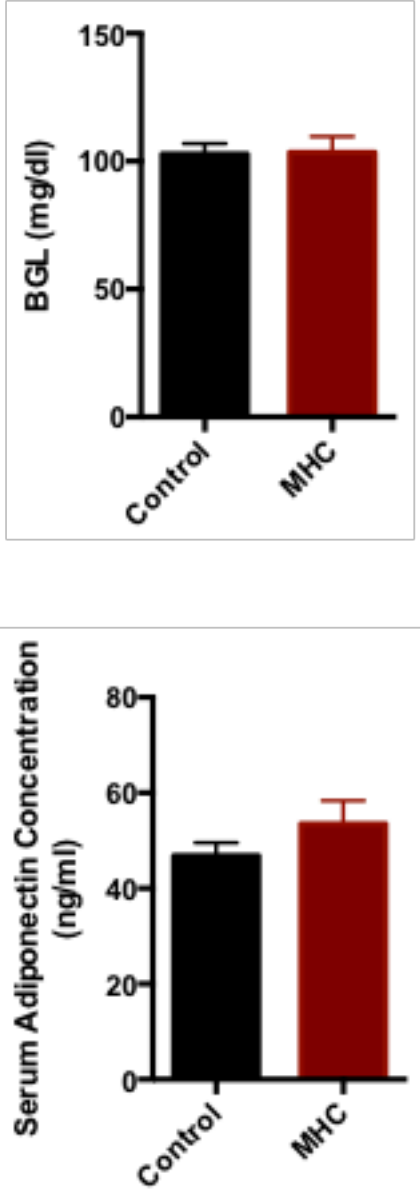
Figure 2

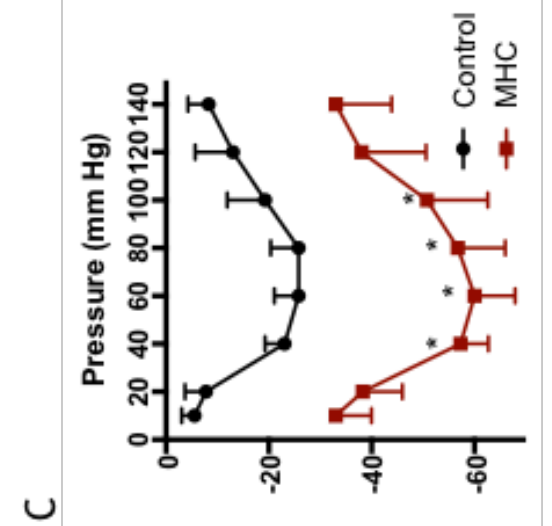

(uni) әนо әмฺฺํㅏ

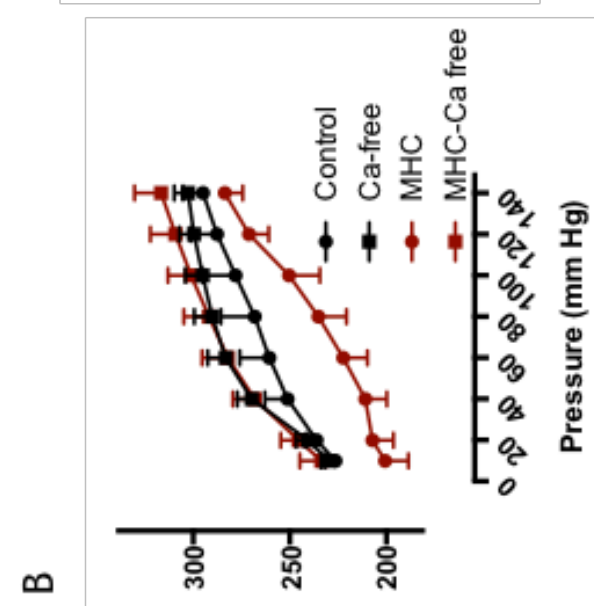

(uri) มәุәแеฺ̣

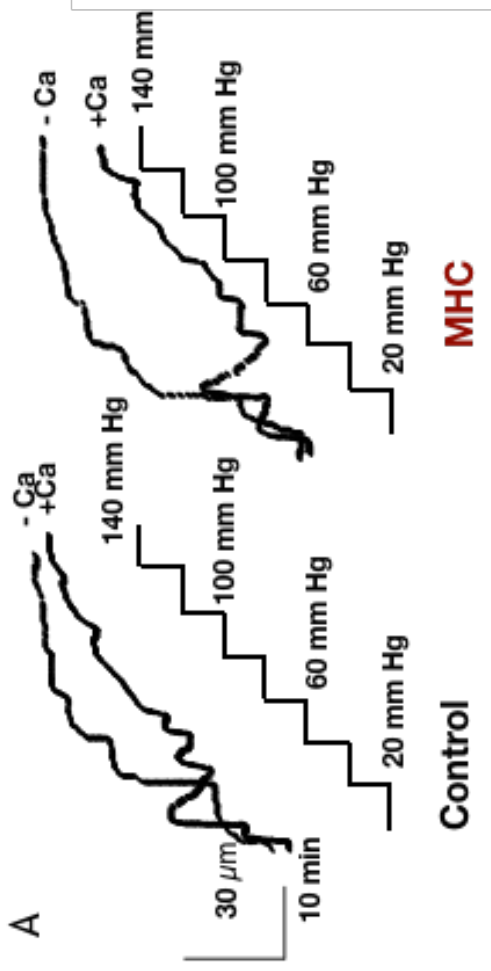

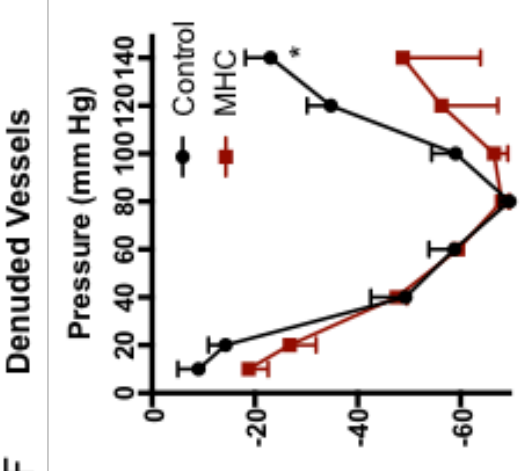

ᄂ

(uni) әนо әм!ฺㅂ

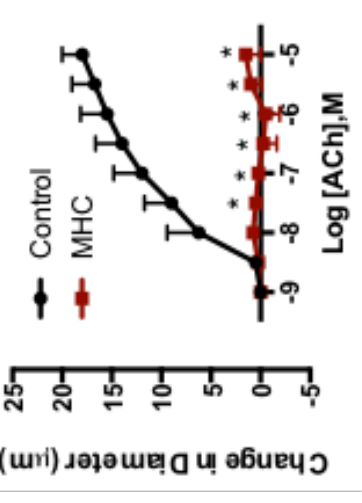

ш
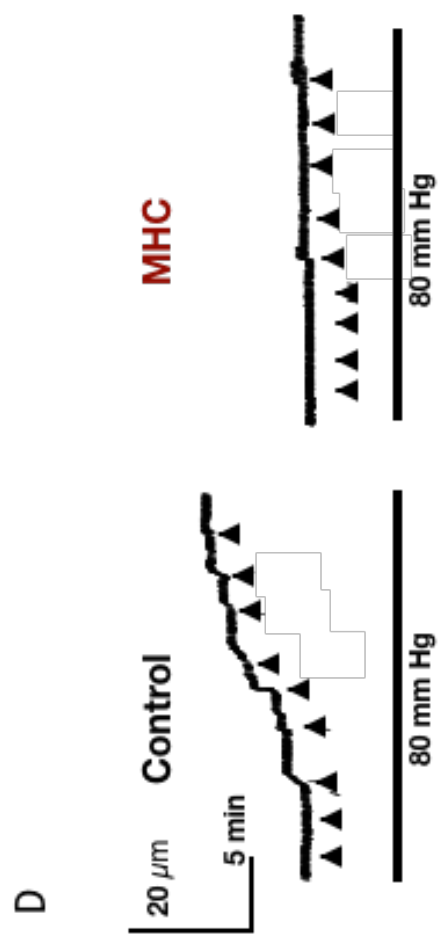
Figure 3
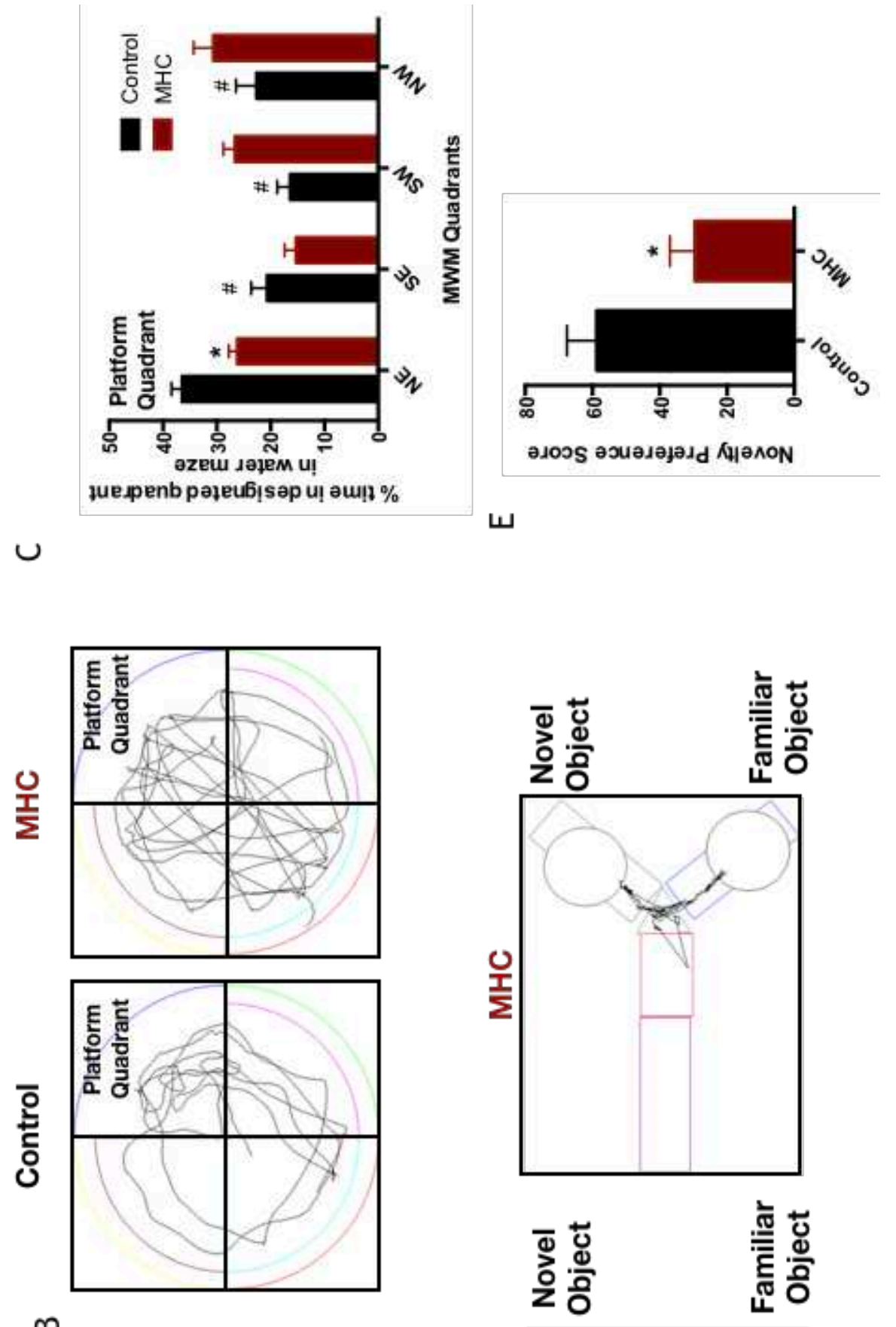

$\infty$

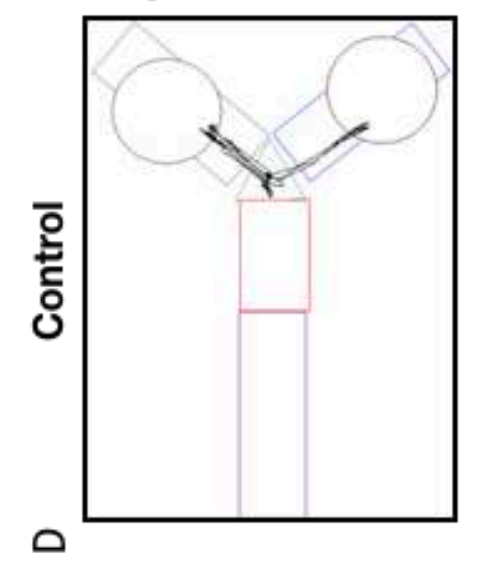


Figure 4

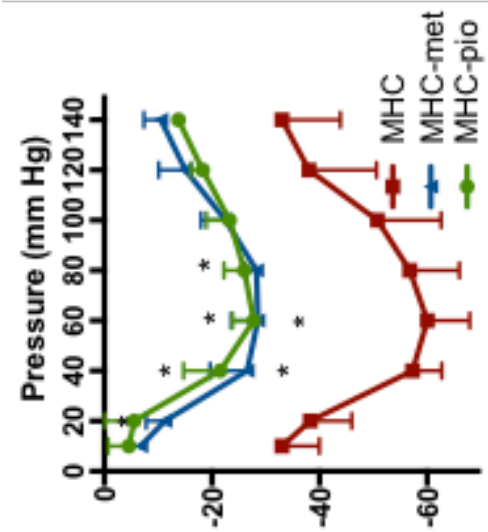

(uri) әนоำ әм!̣ว

$\cup$

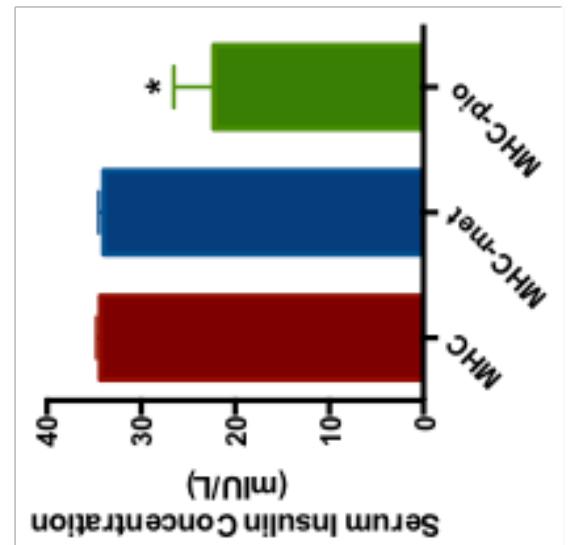

$\infty$

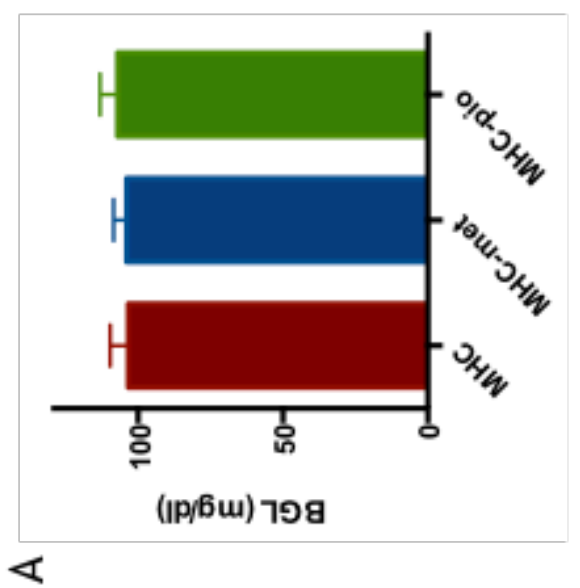

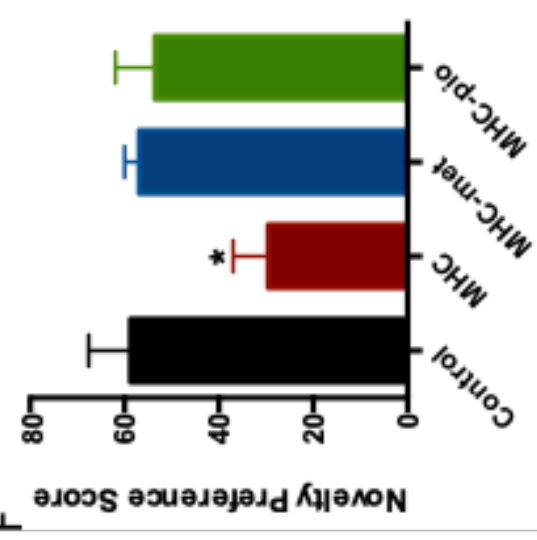

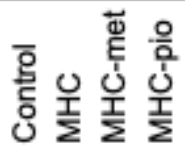

\section{.".}
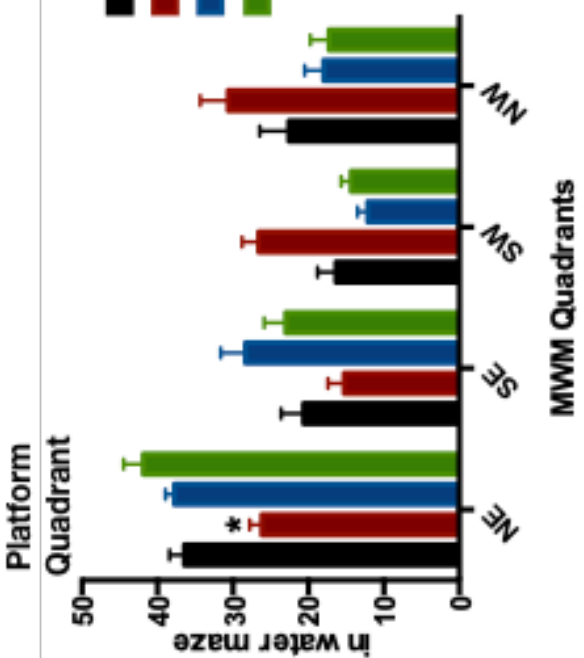

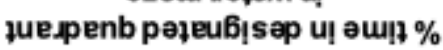

山

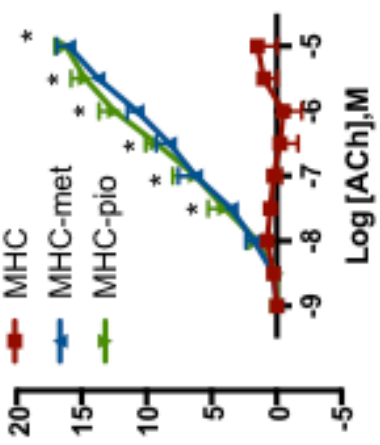

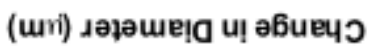

ص 
Figure 5

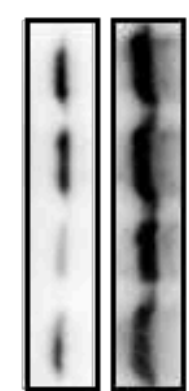

플 은

$\cup$

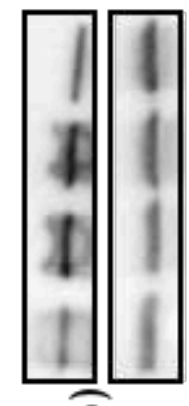

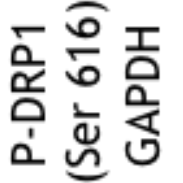

$\infty$

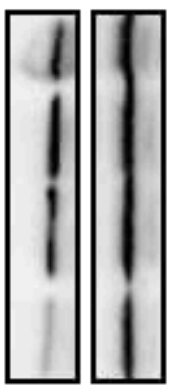

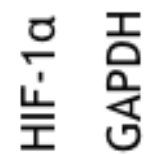

$\varangle$

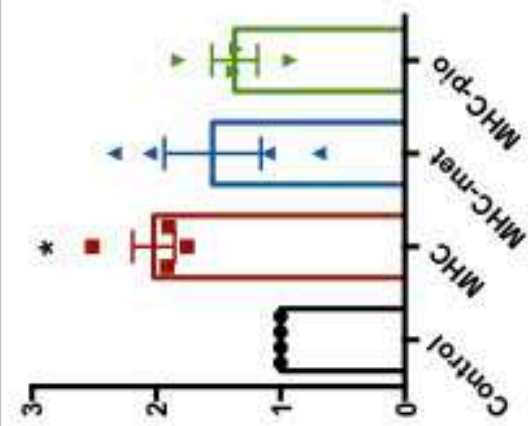

HOd $\forall כ /(9 / 9 \mu a s) ~ เ d y a-d$

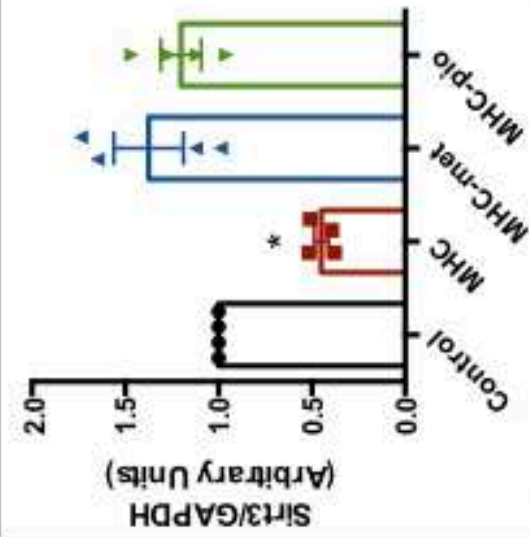

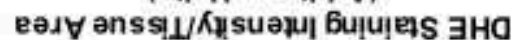

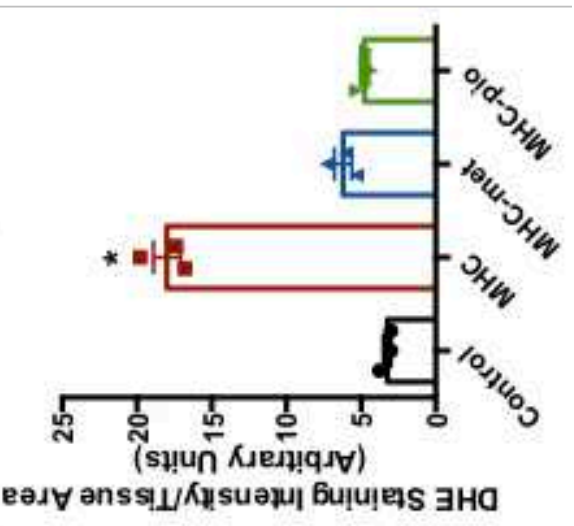

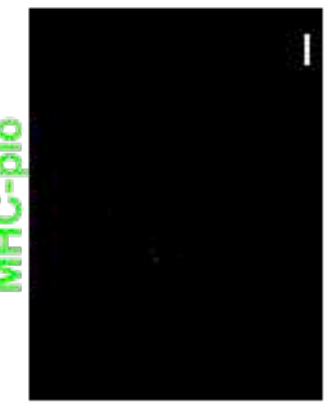
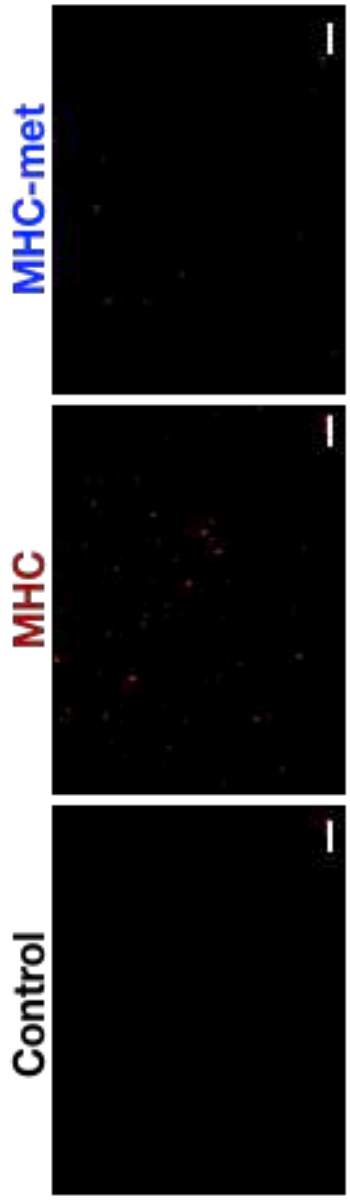

๑ 
Figure 6

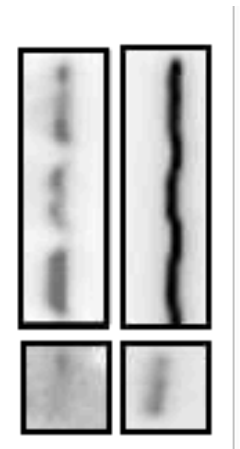

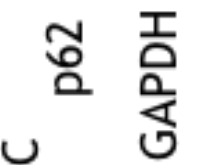

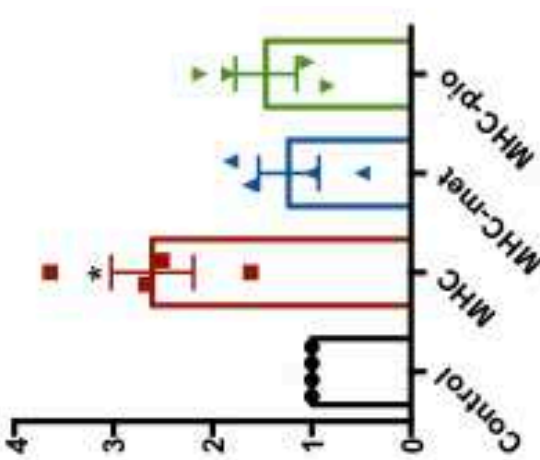

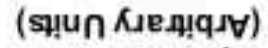
HOdVI/Z9d

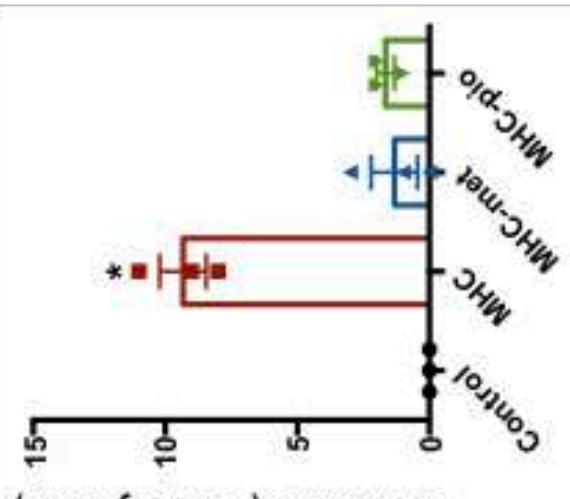

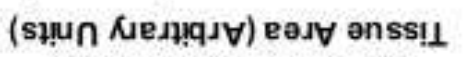
/eวound
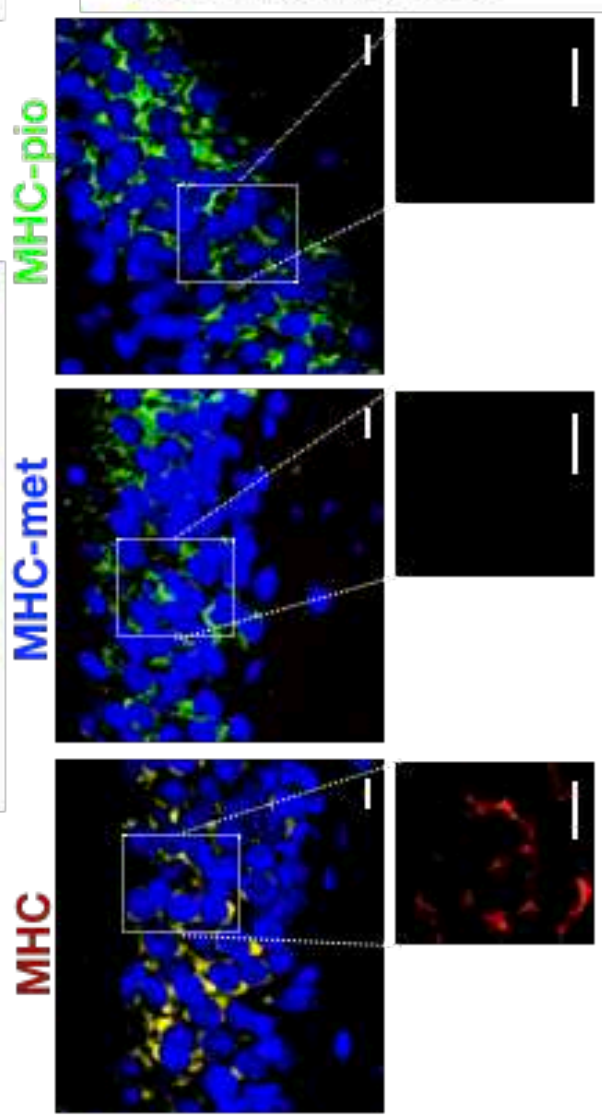

๑
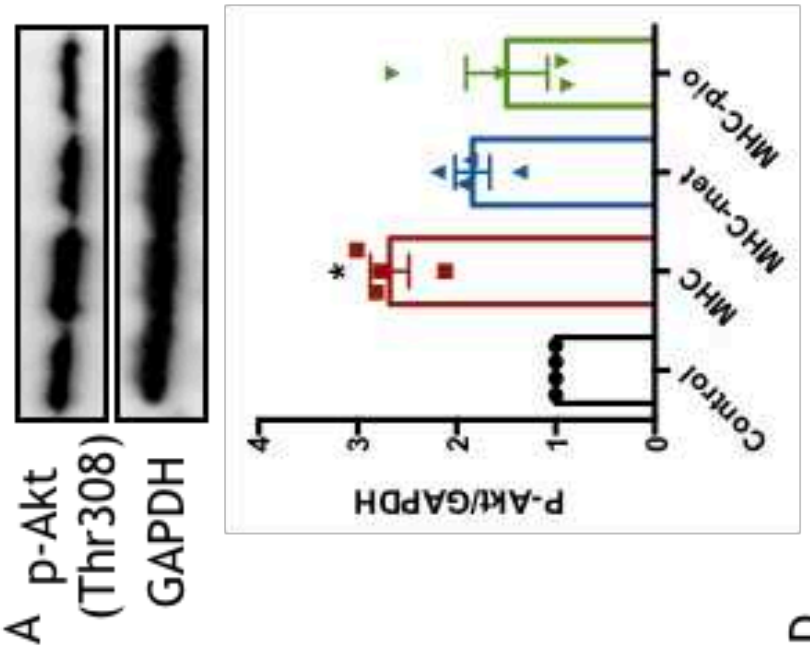

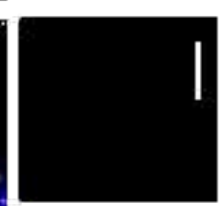

$\stackrel{\oplus}{\text { m }}$

禺
ஹ
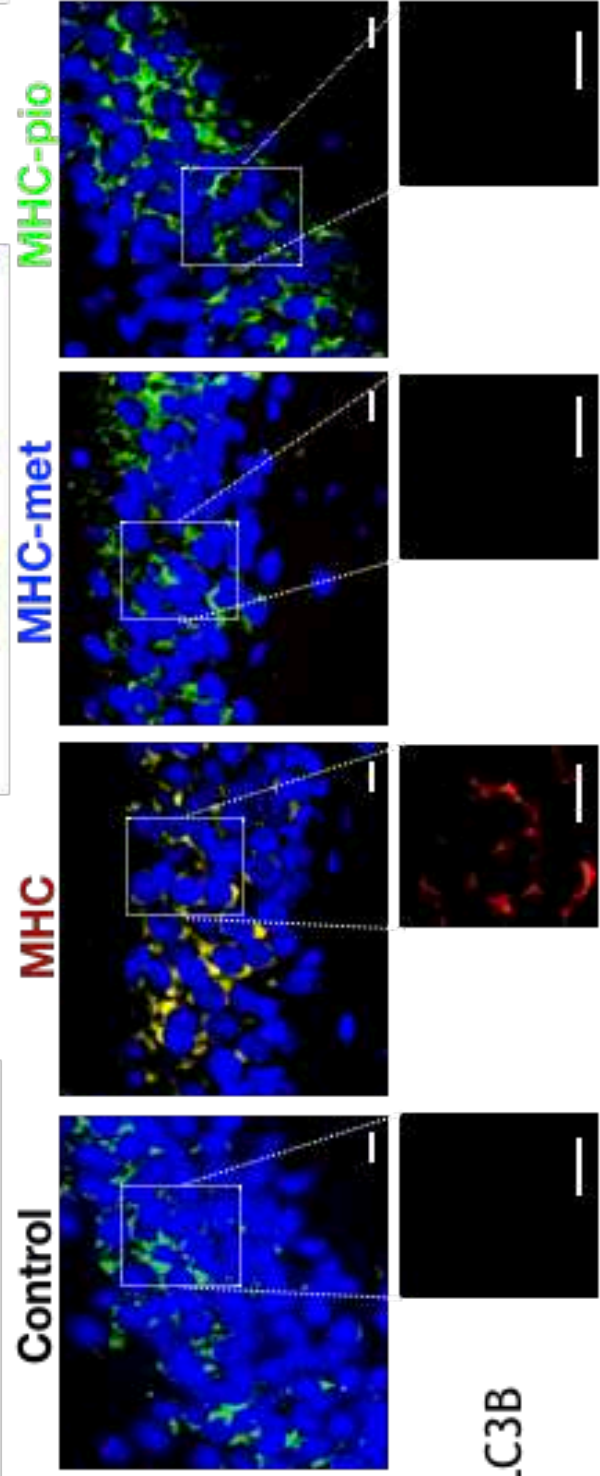

$$
\text { I }
$$

$\Omega$ 
Figure 7

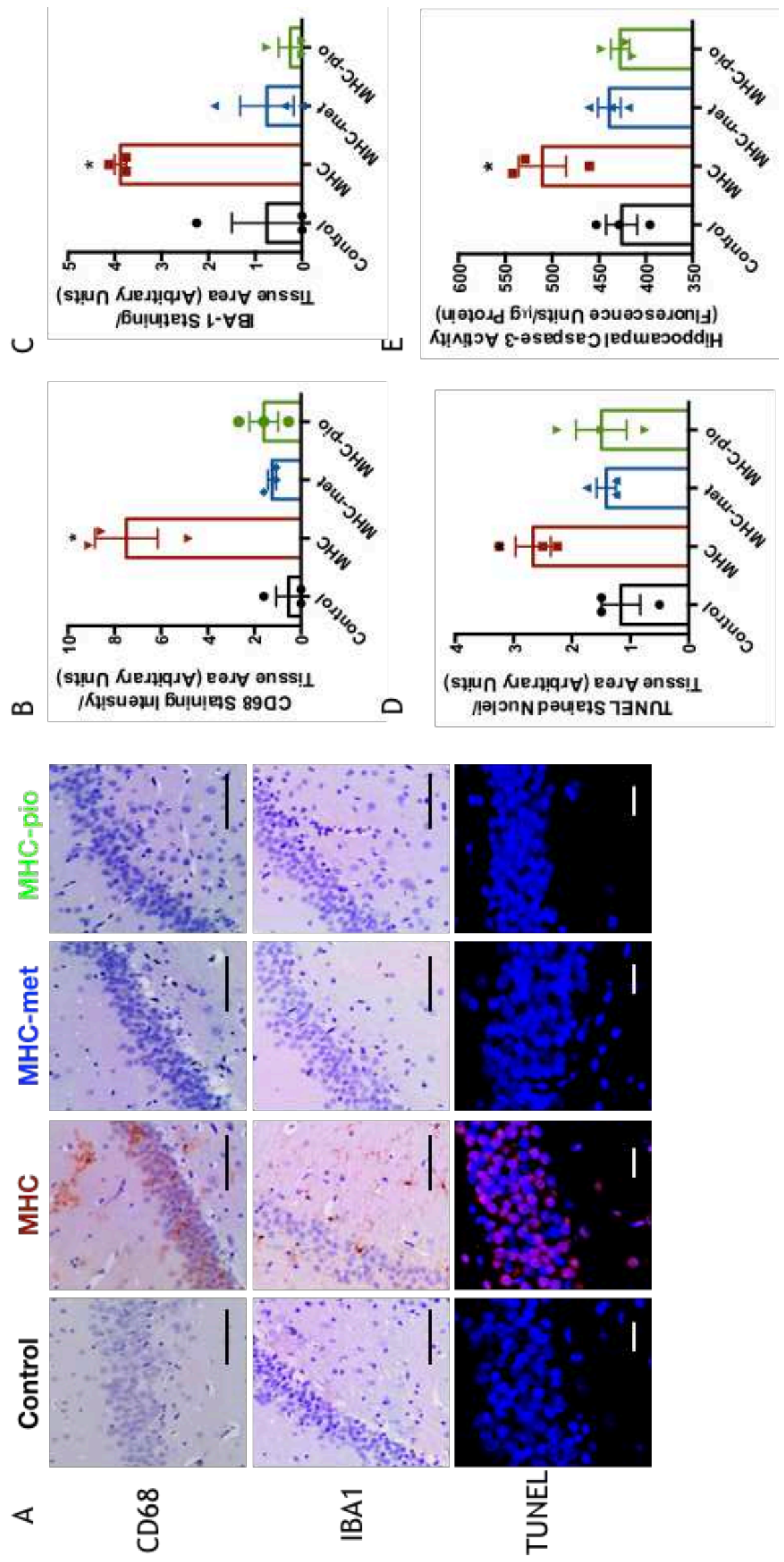

